\title{
DNMT3A harboring leukemia-associated mutations directs sensitivity to DNA damage at replication forks
}

Kartika Venugopal ${ }^{1}$, Pawel Nowialis ${ }^{2}$, Yang Feng ${ }^{1}$, Daniil E. Shabashvili ${ }^{1}$, Cassandra M. Berntsen ${ }^{1}$, Kathryn I. Krajcik ${ }^{1}$, Christina Taragjini ${ }^{1}$, Zachary Zaroogian ${ }^{1}$, Heidi L. Casellas Román ${ }^{1}$, Luisa M. Posada ${ }^{1}$, Chamara Gunaratne ${ }^{1}$, Jianping Li $^{3}$, Daphné Dupéré-Richer ${ }^{3}$, Richard L. Bennett ${ }^{3,7}$, Santhi Pondugula ${ }^{1}$, Alberto Riva ${ }^{4,7}$, Christopher R. Cogle ${ }^{3,7}$, Rene Opavsky ${ }^{2,7}$, Brian K. Law ${ }^{1,7}$, Stefan Kubicek ${ }^{5}$, Philipp B. Staber $^{6}$, Jonathan D. Licht ${ }^{3,7}$, Jonathan E. Bird ${ }^{1}$, and Olga A. Guryanova ${ }^{1,7, *}$

1. Department of Pharmacology \& Therapeutics, University of Florida College of Medicine, Gainesville, FL

2. Department of Anatomy \& Cell Biology, University of Florida College of Medicine, Gainesville, FL

3. Division of Hematology/Oncology, Department of Medicine, University of Florida College of Medicine, Gainesville, FL

4. Bioinformatics Core, Interdisciplinary Center for Biotechnology Research, University of Florida, Gainesville, FL

5. CeMM Research Center for Molecular Medicine of the Austrian Academy of Sciences, Vienna, Austria

6. Division of Hematology and Hemostaseology, Department of Medicine 1, Comprehensive Cancer Center Vienna, Medical University of Vienna, Vienna, Austria

7. University of Florida Health Cancer Center, Gainesville, FL

* Correspondence: Olga A. Guryanova, 1200 Newell Drive, P.O. Box 100267, Gainesville, FL 32610; tel.: +1 (352) 294-8590; email: oguryanova@ufl.edu

Running title: Cells with DNMT3A R882 are sensitive to cytarabine

Keywords: DNMT3A, AML, cytarabine, replication stress, DNA repair

\footnotetext{
\# Abbreviations: AML, acute myeloid leukemia; ara-C, cytosine arabinoside (cytarabine); DDR, DNA damage response; DNMT3A, DNA methyltransferase 3A; DSB, double-strand DNA break; OXPHOS, oxidative phosphorylation; PBS, phosphate buffered saline; SSB, single-strand DNA break; RT, room temperature.
} 


\begin{abstract}
Mutations in the DNA methyltransferase 3A $(D N M T 3 A)$ gene are recurrent in de novo acute myeloid leukemia (AML) and are associated with resistance to standard chemotherapy, disease relapse, and poor prognosis, especially in advanced-age patients. Previous gene expression studies in cells with DNMT3A mutations identified deregulation of cell cycle-related signatures implicated in DNA damage response and replication fork integrity, suggesting sensitivity to replication stress. Here we tested whether pharmacologically-induced replication fork stalling creates a therapeutic vulnerability in cells with DNMT3A(R882) mutations. We observed increased sensitivity to nucleoside analogs such as cytarabine in multiple cellular systems expressing mutant DNMT3A, ectopically or endogenously, in vitro and in vivo. Analysis of DNA damage signaling in response to cytarabine revealed persistent intra-S phase checkpoint activation, accompanied by accumulation of DNA damage in the DNMT3A(R882) overexpressing cells, which was only partially resolved after drug removal and carried through mitosis, resulting in micronucleation. Pulse-chase double-labeling experiments with EdU and BrdU after cytarabine wash-out demonstrated that cells with $D N M T 3 A(m u t)$ were able to restart replication but showed a higher rate of fork collapse. Gene expression profiling by RNA-seq identified deregulation of pathways associated with cell cycle progression and p53 activation, as well as metabolism and chromatin. Together, our studies show that cells with DNMT3A mutations have a defect in recovery from replication fork arrest and subsequent accumulation of unresolved DNA damage, which may have therapeutic tractability. These results demonstrate that, in addition to its role in epigenetic control, DNMT3A contributes to preserving genome integrity during DNA replication.
\end{abstract}




\section{INTRODUCTION}

Acute myeloid leukemia (AML), a genetically heterogeneous malignant clonal disorder of the hematopoietic system, is the most common leukemia in adults ${ }^{1,2}$. Its incidence sharply increases with age, with median age at diagnosis 67-68 years. Due to intensive basic and clinical research, the long-term survival has significantly increased in the last 50 years and is currently approaching $30 \%-40 \%$. Development of targeted therapies has led to remarkable successes in some genetic subtypes of AML leading to improved survival and quality of life. However, for most AML subtypes targeted approaches are still unavailable, leaving cytotoxic chemotherapy the next best option ${ }^{3,4}$. To date, therapeutic targeting for AML with DNMT3A mutations has not shown much promise.

Recurrent mutations in the DNA methyltransferase 3A (DNMT3A) gene are found in $20-35 \%$ of de novo AMLs and are associated with poor $\operatorname{prognosis}^{5-12}$, in part attributable to relative resistance to anthracyclines ${ }^{12-14}$. While chemotherapy dose intensification demonstrated some survival benefit ${ }^{12,14}$, outcomes remain unsatisfactory in most patients with $D N M T 3 A$ alterations due to advanced age, comorbidities, and inability to tolerate treatment ${ }^{1,7,15}$. Cytarabine (Ara-C) remains a foundation of AML treatment $^{16}$, with low-dose Ara-C being a strategy of choice in advanced-age patients ${ }^{3,17-19}$. Clinical trials of low-intensity regimens combining cytarabine and cladribine, nucleoside analog chain terminators that stall DNA replication, with and without venetoclax, are safe and effective $\mathrm{e}^{3,20}$, and in a recent study tended to benefit patients with $D N M T 3 A$ mutations ${ }^{21}$. The most common mutation type, representing up to $70 \%$ of all DNMT3A alterations in AML, is single amino-acid substitution at arginine 882 (R882, most commonly $\mathrm{R} 882 \mathrm{H}$ or $\mathrm{R} 882 \mathrm{C}$ ) in the catalytic domain ${ }^{9,11,12,22,23}$. Hence, we and others performed DNA methylation analyses $^{13,24-27}$ and gene expression profiling ${ }^{13,28}$ in primary AML samples and in animal models carrying DNMT3A mutations. These studies uncovered gene signatures of altered cell cycle control such as negative enrichment of the CHK1-regulated G2/M checkpoint ${ }^{29,30}$, which may indicate persistent replication stress, in cells expressing mutant $D N M T 3 A^{13,28}$. Importantly, DNMT3A protein has been detected in direct 
association with stalled replication forks in cells treated with hydroxyurea ${ }^{31}$. Yet, its role in the context of replication fork stalling such as after cytarabine-based chemotherapy remains unknown.

Once cytarabine is converted into its active metabolite ara-CTP, it is incorporated into nascent DNA during replication where it is a poor substrate for chain extension ${ }^{32}$. This results in chain termination, replication fork stalling, and DNA damage response (DDR) often leading to p53-dependent apoptosis ${ }^{33-36}$. Singlestranded DNA breaks (SSBs) resulting from stalled replication forks actuate ATR-dependent CHK1 phosphorylation necessary to preserve replication fork integrity ${ }^{37}$. If unresolved, SSBs are converted to double-stranded DNA breaks (DSBs) and activate ATM-dependent phosphorylation cascade of CHK2, H2A.X, and p53, which in turn mediate DNA DSB repair, and cell cycle arrest and/or apoptosis ${ }^{38-40}$.

This study investigated the role of mutant DNMT3A (DNMT3A ${ }^{\text {mut }}$ ) in recovery of stalled replication forks and therapeutic responses to replication stress-inducing drugs. We show in multiple systems that cells expressing $D N M T 3 A^{m u t}$ are more sensitive to cytarabine-induced replication. This was accompanied by persistent intra-S checkpoint activation and accumulation of unrepaired DNA damage, which was carried through mitosis, leading to mitotic defects ${ }^{41}$. Mechanistic studies of replication fork dynamics in cytarabinetreated cells demonstrated a higher rate of replication fork collapse in cells with DNMT3A ${ }^{\text {mut }}$. These results provide mechanistic insights into the regulation of replication fork recovery after DNA damage, and suggest additional strategies to augment therapeutic responses to cytarabine and similar drugs used to treat AML. 


\section{MATERIALS AND METHODS}

Cell lines: Human leukemia cell lines K-562, KU-812, SET-2, and KO-52 were grown in RPMI supplemented with 10\% FBS and penicillin/streptomycin. U2OS cells were maintained in DMEM supplemented with $4.5 \mathrm{~g} / 1$ glucose, nonessential amino acids, 10\% FBS, and penicillin/streptomycin. Cells were lentivirally transduced to express wild-type or R882C-mutant DNMT3A or with empty vector control (pMIGR1 expressing GFP as a selectable marker, or pPICH expressing dsRED). For robustness, all experiments were performed with both sets of isogenic cell lines, one with pMIGR1 and one with pPICH, as independent biological replicates.

Cell viability and drug dose response assays: Cells were plated in 96 -well plates at $1 \times 10^{4} /$ well (suspension) and $3 \times 10^{3} /$ well (adherent) and exposed to cytarabine (Ara-C), fludarabine, or cladribine (Cayman Chemicals), or hydroxyurea (HU) (Sigma) in triplicate. Relative cell numbers were quantified at the indicated time points by CellTiter Glo (Promega) for suspension cultures or by alamarBlue (Thermo Fisher) for adherent cells. Plates were read with a BMG LABTECH FluoroQuant Optima plate reader. Relative cell viability data normalized to vehicle controls set at $100 \%$ were used to calculate $\mathrm{IC}_{50}$ values by fitting a four-parameter (variable slope) dose-response curve. GraphPad Prism version 7 software was used.

Mice: All animal studies were approved by the University of Florida Institutional Animal Care and Use Committee. A knock-in mouse line that conditionally expresses Dnmt $3 a^{R 878 H}$ allele (equivalent to $D N M T 3 A^{R 882 H}$ in humans) from the endogenous locus (Jackson Laboratory stock No. 031514) was described $^{13}$. For inducible expression of the mutant form of Dnmt $3 a$ and generation of mouse leukemias with Flt $3^{I T D}$ and $N p m 1^{c}$ alleles see Supplementary methods. For in vivo cytarabine treatment studies, preconditioned congenic CD45.1 recipients were transplanted with CD45.2 donor bone marrow from fully

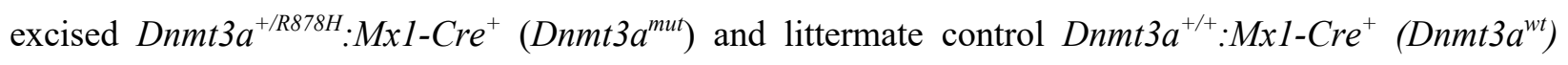
mice. Once engrafted, animals were treated with $30 \mathrm{mg} / \mathrm{kg}$ cytarabine/day for 5 days IP and vehicle (PBS) 
( $n=5 /$ group). Bone marrow was analyzed after $48 \mathrm{~h}$. See also Supplementary methods.

Colony forming unit (CFU) assays: Freshly isolated whole bone marrow cells harvested from the Dnmt3a wildtype and mutant mice (described above) were plated in MethoCult M3434 (StemCell), $1 \times 10^{4}$ cells/well in triplicate. Vehicle (PBS) or cytarabine were added directly to the methylcellulose media at indicated concentrations. Colonies were scored 10-14 days after plating and imaged by EVOS FL microscope using a $2 \times$ objective.

Immunoblotting: Ara-C-treated cells were lysed in RIPA buffer supplemented with protease and phosphatase inhibitors, and $25 \mu \mathrm{g}$ of total protein were resolved by SDS-PAGE, blotted onto PVDF membranes (Millipore), probed by standard methods, and signals detected by chemoluminescence as described in Supplementary Methods. Primary antibodies are listed in Supplementary Table S1. For preparation of chromatin-bound proteins and soluble nuclear extracts, treated cells were trypsizined, washed in ice-cold PBS, and $2 \times 10^{6}$ cells were processed using Subcellular Protein Fractionation Kit for cultured cells (Pierce) according to manufacturer's instructions, and analyzed by Western blotting.

Cell cycle analysis and intracellular flow cytometry: Cell cycle was analyzed based on DNA content by 4',6-diamidino-2-phenylindole (DAPI) staining as described in Supplementary methods. Primary antibodies are listed in Supplementary Table S2.

Replication restart by dual labeling with EdU and BrdU: Cells in culture were pulsed with EdU $(10 \mu \mathrm{M})$ (C10643, Invitrogen) for 30 mins. Plates were then washed and treated with $10 \mu \mathrm{M}$ of Ara-C for 12 hours,

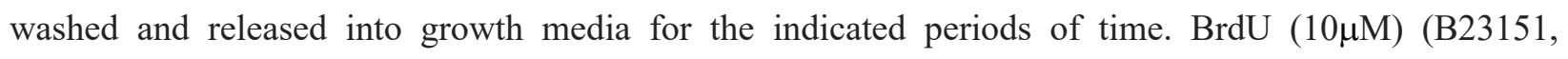
Invitrogen) was added 30 minutes before harvesting. Trypsinized cells were fixed with $70 \%$ ethanol at $4{ }^{\circ} \mathrm{C}$ overnight, incubated in $2 \mathrm{M} \mathrm{HCl}$ solution for 30 minutes at RT, and washed in 3\% BSA in PBS. EdU was 
detected using Click-iT EdU Alexa647 picolyl-azide toolkit (C10643, Invitrogen) according to the manufacturer's protocol. Next, cells were incubated with mouse anti-BrdU monoclonal antibody conjugated to Alexa Fluor 488 (1:20, B35130, Invitrogen) in antibody staining solution (1\% BSA, 0.2\% Tween-20 in PBS) for 1 hour at RT. After washing in PBS, cells were incubated with DAPI and analyzed by flow cytometry.

Comet Assay: Alkaline comet assays were performed using CometSlides and Comet Assay kit (Trevigen). See Supplementary methods.

Immunofluorescence analysis: U2OS cells were seeded $2.5 \times 10^{4}$ cells/well on $12 \mathrm{~mm}$ coverslips in 12 well plates, allowed to attach overnight, and treated as indicated. Cells were fixed in $2 \%$ paraformaldehyde in PBS for 30 minutes at RT, washed with PBS, permeabilized by $0.5 \%$ Triton X-100 in PBS for 10 minutes at RT, and washed with $1 \mathrm{mM}$ glycine to quench unreacted aldehydes. Cells were incubated in blocking buffer (0.1\% BSA, $0.05 \%$ Tween-20, 0.2\% Triton X-100, 10\% donkey serum) for 1 hour at RT. Coverslips were incubated with primary antibodies specific to $\gamma \mathrm{H} 2 \mathrm{~A} . \mathrm{X}$, PCNA, pRPA (see Supplementary Table S3) at manufacturer recommended dilutions in blocking buffer overnight at $4^{\circ} \mathrm{C}$. After washing in PBS, cells were incubated with AlexaFluor488 or AlexaFluor594-conjugated donkey anti-mouse or anti-rabbit secondary antibodies (Invitrogen) (1:1000) in blocking buffer for 1 hour at RT and washed twice in PBS. Coverslips were mounted using ProLong Gold medium with DAPI (Invitrogen). Slides were examined using a spinning disc confocal (Yokogawa CSU-X1) attached to an inverted microscope (Nikon Eclipse Ti2-E) using a 60× objective (Nikon Plan Apo, 1.4 NA). For quantitative analyses, at least 50 nuclei were selected at random by thresholding based on the DAPI channel, and background-corrected integrated fluorescence density was calculated using ImageJ software. For BrdU and EdU immunofluorescence analysis, and analysis of micronucleation and nuclear morphology see Supplementary methods. 
Metaphase spreads: U2OS cells were treated with $10 \mu \mathrm{M}$ Ara-C for 12 hours, washed in PBS and released in complete media for 16 hours. The cells were incubated in 50ng/ml colcemid (KaryoMAX, GIBCO) for 12 hours, harvested and swelled with a hypotonic solution $(75 \mathrm{mM} \mathrm{KCl})$ for 15 minutes at $37^{\circ} \mathrm{C}$. The cells were then fixed in ice-cold 3:1 mixture of methanol/acetic acid and dropped on slides. Slides were stained with 5\% Giemsa (Sigma), rinsed with distilled water, coverslipped using Permount (Fisher), and scanned on a Keyence BZ-X800 microscope with a 40× NA 0.95 objective.

RNA-Seq: RNA integrity and purity were assessed by Qubit and Agilent 2100 Bioanalyzer (Agilent Technologies, Santa Clara, CA), and high-quality samples with RIN $>8.0$ were sent to Novogene Co. Ltd for library preparation and sequencing on an Illumina NovaSeq 6000 using paired-end 150 chemistry. Sequencing data QC, alignment, quantification, differential expression, and pathway analysis were performed by standard methods as described in Supplementary methods.

Data sharing: Raw and processed RNA-seq data are available at GEO under the accession code GSE153871.

Statistical analysis and rigor: Groups were compared using Student's $t$-test for normally distributed data and Mann-Whitney rank sum test for non-normally distributed data, after testing for normality and equal variance; $p$-values $\leq 0.05$ were considered significant. Where appropriate, one-way ANOVA with Tukey's HSD post-hoc test or two-way ANOVA with post-hoc Š́dák correction for multiple hypothesis testing were performed. Data were plotted as mean \pm standard deviation (SD) unless indicated otherwise. For categorical data, two-tailed Fisher's exact test was applied. GraphPad Prism software version 7 or higher was used. Identity of cell lines was validated by STR profiling; cultures were routinely tested for Mycoplasma contamination. Image scoring was done blindly; investigators were unblinded after data were collected. In vivo studies were repeated twice with comparable results. All other experiments were independently performed three or more times for reproducibility. 


\section{RESULTS}

\section{Cells with $D N M T 3 A$ R882 mutations are more sensitive to replication-stalling drugs}

Our prior studies found that $D N M T 3 A$-mutant cells were relatively resistant to anthracyclines, and showed no differential sensitivity to DNA cross-linking and DSB inducing agents ${ }^{13}$. To investigate the role of DNMT3A in replication stress sensitivity, we treated a panel of leukemia cell lines with and without $D N M T 3 A^{R 882}$ mutations with the cytosine analog cytarabine and purine analog fludarabine (Fig. 1A,B), purine analog cladribine, and hydroxyurea $(\mathrm{HU})$ which stalls replication by depleting deoxyribonucleotides (Supplementary Fig. S1A,B). Cell lines with DNMT3A $A^{R 82}$ SET-2 and KO-52 were more sensitive to replication-stalling drugs than $D N M T 3 A^{W T}$ cells K-562 and KU-812, reflected by increased apoptosis measured by annexin $\mathrm{V}$ and the cell fraction with sub-G $\mathrm{G}_{1} \mathrm{DNA}$ content (Supplementary Fig. S1C,D).

We extended these observations to normal and leukemic murine hematopoiesis. Freshly isolated bone marrow cells from mice with conditional Dnmt $3 a^{R 878 H}$ expression (corresponding to human $D N M T 3 A^{R 882 H}$ ) plated in the presence of increasing Ara-C concentrations demonstrated a dampened clonogenic potential ex vivo in semisolid media compared to wild-type controls (Fig. 1C). Additionally, in a mouse model of AML driven by $F l t 3^{I T D}$ and $N p m 1^{c}$, bone marrow cells from leukemic animals with $D n m t 3 a^{R 878 H}$ were more sensitive to cytarabine than Dnmt3a $a^{W T}$ in liquid culture ex vivo (Fig. 1D). In mice reconstituted with Dnmt $3 a^{R 878 H}$ but not WT control bone marrow, administration of cytarabine over 5 days in vivo led to significant depletion of peripheral blood myeloid $\left(\mathrm{CD}_{11 \mathrm{~b}^{+}}\right)$cells and a compensatory increase in B-cells $\left(\mathrm{B} 220^{+}\right)$, along with elevated apoptosis of hematopoietic stem and progenitor-enriched LSK cell population (Fig. 1E-G). Finally, in primary bone marrow samples from AML patients harboring $F L T 3^{I T D}$ and $N P M 1^{c}$ mutations, presence of $D N M T 3 A^{R 882}$ was associated with increased ex vivo sensitivity to Ara-C compared to $D N M T 3 A^{W T}$ (Supplementary Fig. S1E). Together, these data indicate that expression of mutant $D N M T 3 A$ in hematopoietic cells is associated with increased sensitivity to pharmacologically-induced replication stress. 
A

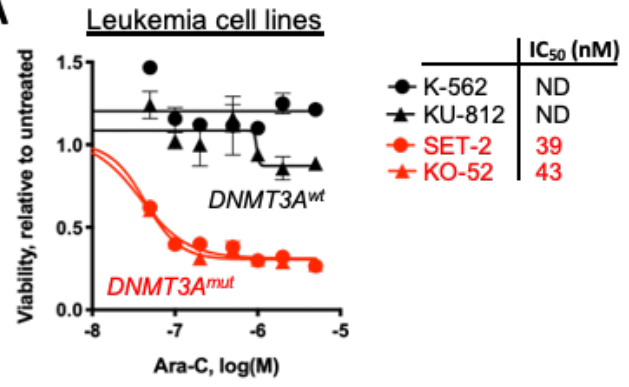

C

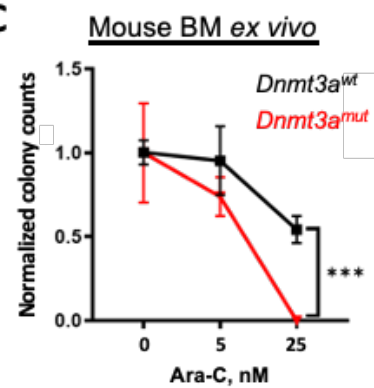

$\mathbf{E}$

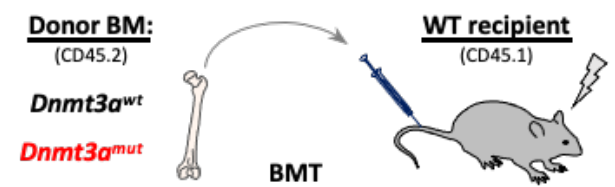

$\mathbf{F}$

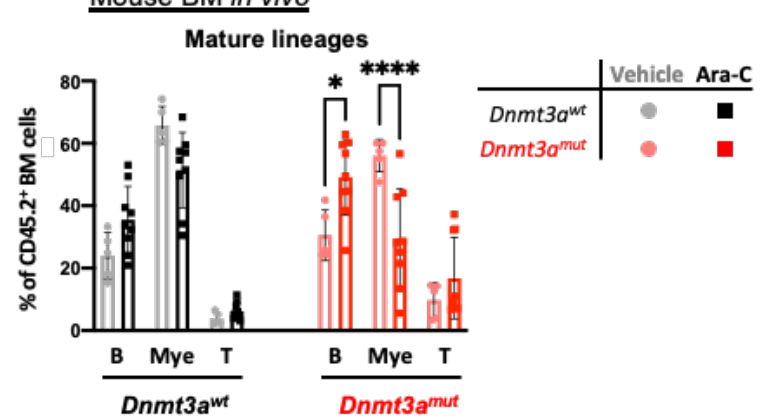

H

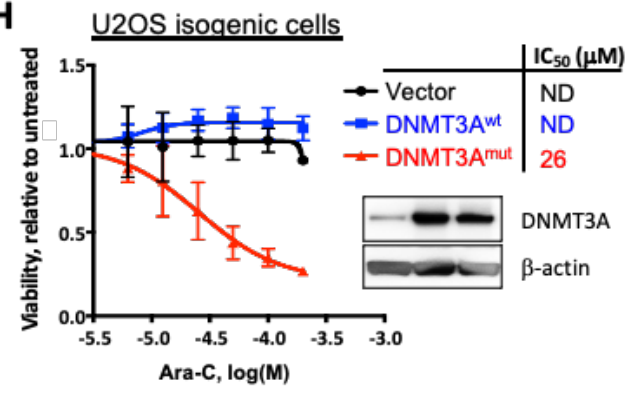

B Leukemia cell lines

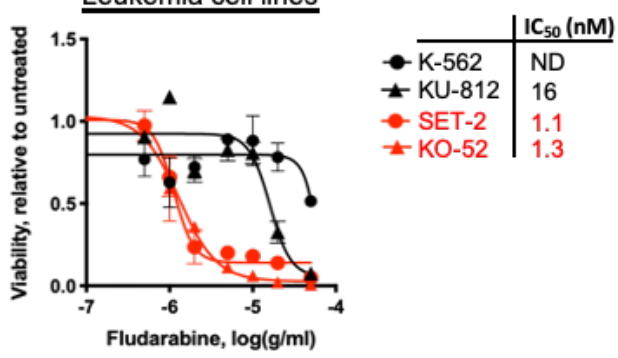

D Mouse BM ex vivo
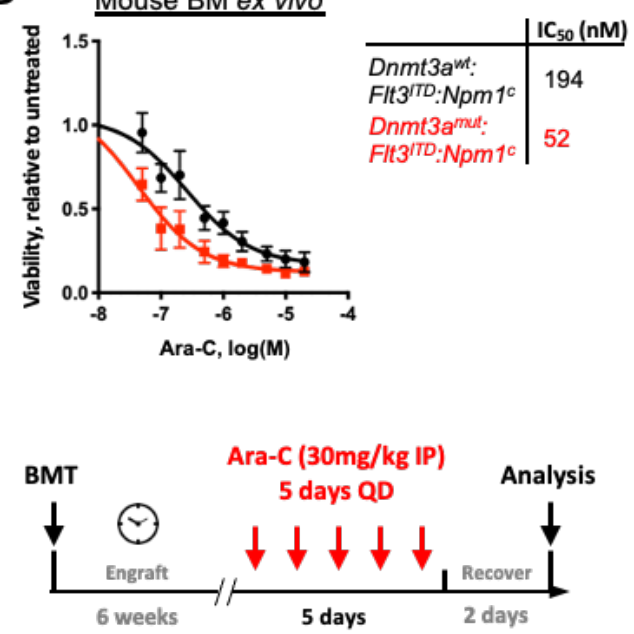

G

Mouse BM in vivo

Apoptosis

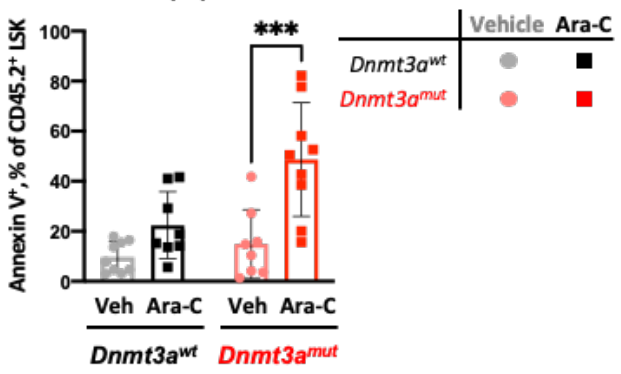

I

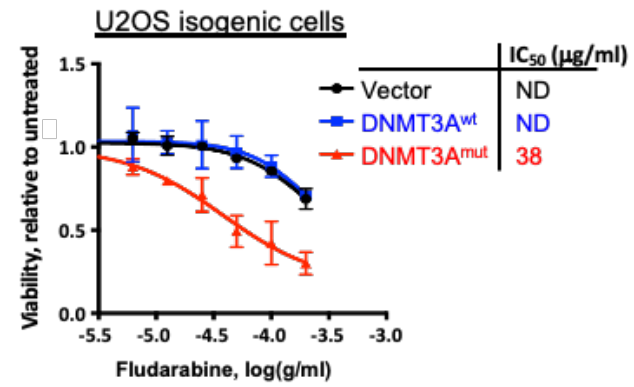

Figure 1. Expression of mutant DNMT3A confers sensitivity to replication stalling agents. (A, B) Sensitivity to elongation-terminating nucleoside analogs cytarabine (Ara-C, A) and fludarabine (B) and corresponding $\mathrm{IC}_{50}$ values 
in leukemia cell lines with $D N M T 3 A^{R 882}$ (red, SET-2 and KO-52) and DNMT3A $A^{W T}$ (black, K-562 and KU-812) after $48 \mathrm{~h}$ of treatment, relative to vehicle control, as determined by CellTiter GLO assay in triplicate. (C) Colony formation assay using bone marrow cells derived from $D n m t 3 a^{R 878 H}$ and littermate Dnmt $3 a^{w t}$ control mice, treated with indicated concentrations of Ara-C and grown in MethoCult M3434, in triplicate. Colonies were scored 10 days after plating and counts normalized to vehicle controls (C). Bone marrow from Dnmt $3 a^{m u t}$ mice formed fewer to no colonies compared to wildtype control when treated with $25 \mathrm{nM}$ cytarabine; cDNA Sanger sequencing traces show complete Cremediated excision resulting in equal expression of $w t$ and $R 878 H$ alleles. (D) Ex vivo cytarabine dose responses and corresponding $\mathrm{IC}_{50}$ of mouse leukemias driven by $F l t 3^{I T D}: N p m 1^{c}$, with and without Dnmt $3 a^{m u t}$, as measured by CellTiter GLO assay in triplicate. (E-G) Sensitivity to cytarabine in vivo. Congenic CD45.1 recipients reconstituted with $D n m t 3 a^{m u t}$ or wild-type control bone marrow cells received 5 daily doses of $30 \mathrm{mg} / \mathrm{kg}$ Ara-C and were analyzed 48 hours later (E). Frequencies of donor-derived (CD45.2 $2^{+}$mature lineage cells (myeloid, B-cells, T-cells) (F) and apoptosis (Annexin V positivity) of the LSK cells (G) in the bone marrow 48 hours after last treatment dose $(n=5-9$, $* p<0.05, * * * p<0.001, * * * * p<0.0001$, two-way ANOVA with Š́dák's post-hoc multiple comparisons test). (H, I) Sensitivity to cytarabine $(\mathrm{H})$ and fludarabine (I) and corresponding $\mathrm{IC}_{50}$ values in U2OS cells ectopically expressing wild-type (blue) and R882C mutant (red) forms of DNMT3A or empty vector control (black), relative to untreated after $48 \mathrm{~h}$ of treatment, by AlamarBlue assay. Inset in $(\mathrm{H})$ shows levels of expression of wild-type and mutant DNMT3A, and endogenous DNMT3A in cells transduced with an empty vector control.

\section{Cells expressing mutant $D N M T 3 A$ accumulate unresolved DNA damage after cytarabine treatment}

To investigate the molecular mechanism of sensitivity to replication-stalling drugs, we lentivirally transduced wild-type and R882C-mutant forms of DNMT3A into U2OS cells, a well-established model for DNA damage and repair studies, and confirmed differential cell killing by cytarabine and fludarabine (Fig. 1H,I, and Supplementary Fig. S1F,G). At the same time, steady-state U2OS cell growth was unaffected (Supplementary Fig. S1H).

We next investigated the DNA damage response to cytarabine-induced (approx. $\mathrm{IC}_{20}$ ) replication fork stalling in cells with $D N M T 3 A^{R 882 C}$. Cells overexpressing mutant DNMT3A showed CHK1 phosphorylation persisting over 24 hours of continuous drug exposure and a concomitant increase in p53 phosphorylation, while in $D N M T 3 A^{W T}$-expressing cells there was a rapid decline in pCHK1 and a less pronounced p53 activation (Fig. 2A). The intensified signaling in $D N M T 3 A^{m u t}$-expressing cells reflected accumulation of DNA damage as measured by an increased fraction of mobile DNA per nucleus in an alkaline comet assay

(Fig. 2B,C) and confirmed by immunofluorescent detection of phosphorylated histone H2A.X ( $\gamma \mathrm{H} 2 \mathrm{~A} . \mathrm{X})$ (Supplementary Fig. S2A,B). Consistently, SET-2 and KO-52 cell lines with $D N M T 3 A^{R 882}$ demonstrated accumulation of $\gamma \mathrm{H} 2 \mathrm{~A} . \mathrm{X}$ and cleaved PARP indicative of apoptosis along with persisting low-level pCHK1, while in DNMT3A $A^{W T}$ K-562 and KU-812 cells CHK1 activation was rapidly resolved 
A

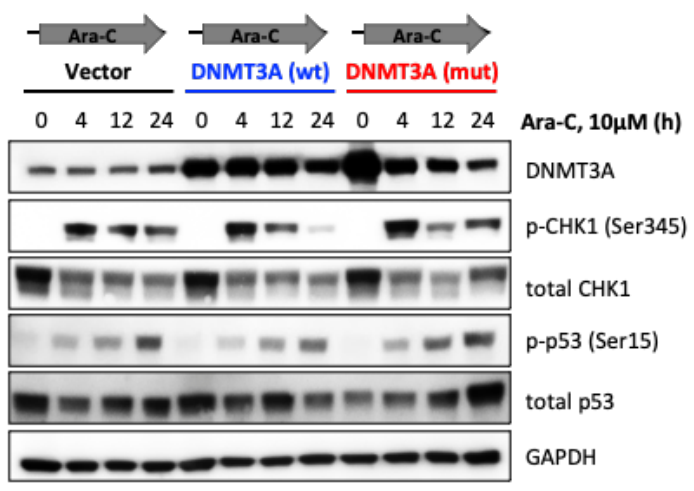

B
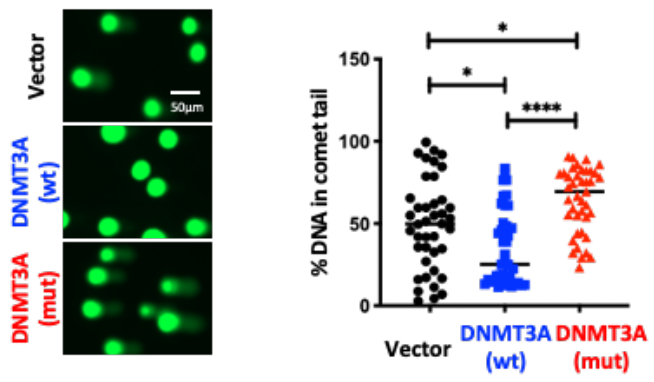

C

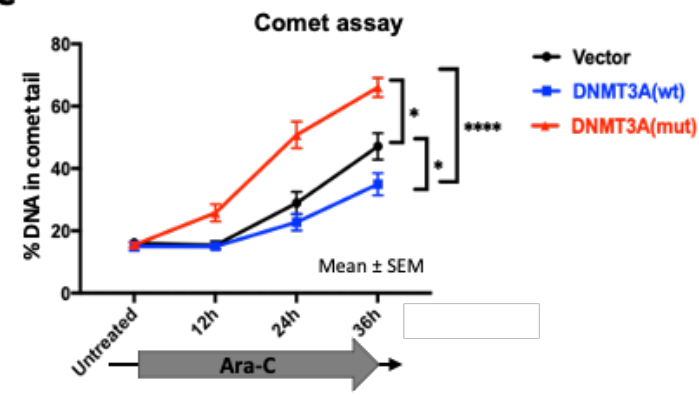

D

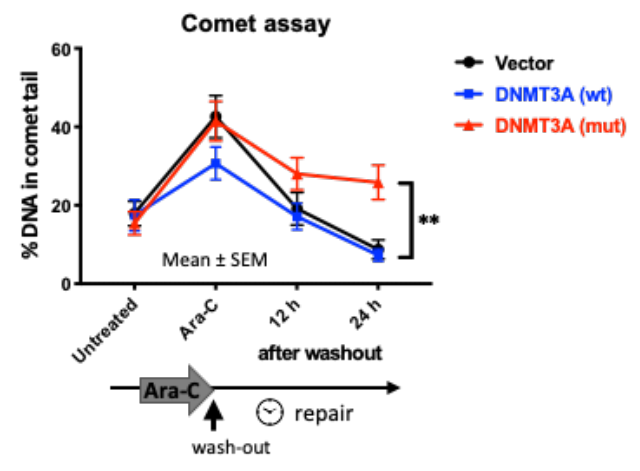

E

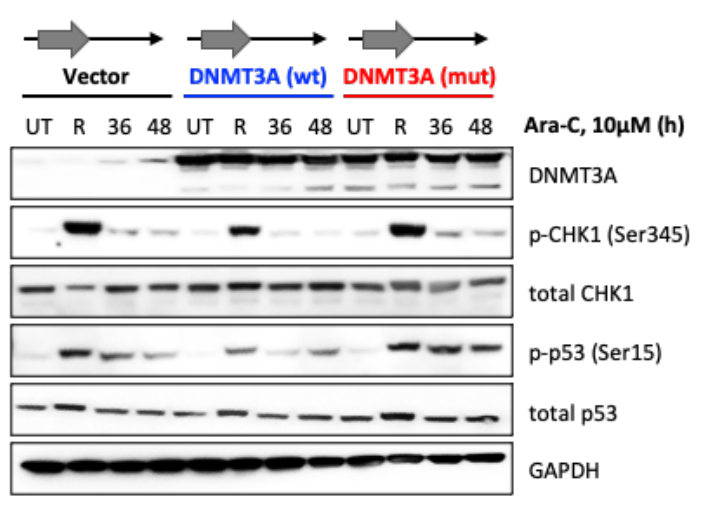

$\mathbf{F}$

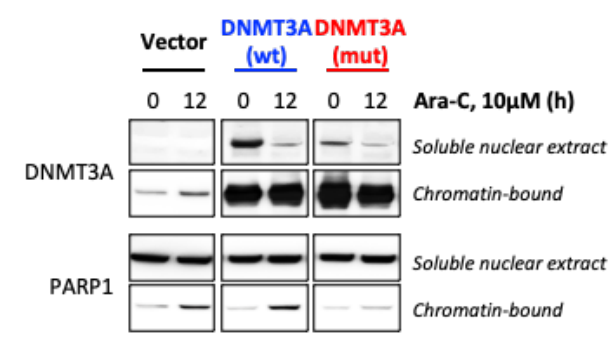

Figure 2. Cells expressing mutant DNMT3A accumulate DNA damage due to incomplete DNA repair upon cytarabine treatment. (A) Immunoblot analysis of the DNA damage signaling at indicated timepoints after cytarabine treatment in U2OS cells with wild-type and mutant DNMT3A or empty vector control. (B, C) Detection of damaged DNA by alkaline Comet assay at 24 hours (B) or at indicated timepoints of continuous cytarabine exposure (C) in U2OS cells lentivirally expressing wild-type (blue) and mutant (red) DNMT3A or empty vector control (black). (D) Incomplete DNA repair in cells expressing mutant DNMT3A 12 and 24 hours after cytarabine removal following $12 \mathrm{~h}$ treatment. At least 40 comets per condition were scored using OpenComet plugin for ImageJ software (NIH) by calculating percentage of DNA in the comet tail on the basis of comet head and tail integral intensity. $(* p<0.05$, $* * p<0.01, * * * * p<0.0001$, Mann-Whitney rank-sum test; graphs represent mean $\pm \mathrm{SEM}$ ). (E) Analysis of DNA damage response in U2OS cells lentivirally expressing wild-type and mutant DNMT3A or empty vector control at 12 hours of cytarabine exposure and 36 and 48 hours after drug wash-out (UT, untreated; R, at release). (F) Recruitment of PARP1 to chromatin from soluble nuclear extract after cytarabine treatment in cells overexpressing wild-type or mutant forms of DNMT3A.

(Supplementary Fig. S2C). These results indicate that cells with mutant $D N M T 3 A$ accumulate DNA damage 
after cytarabine.

Deficiency in DNA repair is a common explanation for accumulation of DNA damage in drug treated cells. Accordingly, DNA damage resolution after cytarabine wash-out was markedly delayed in $D N M T 3 A^{R 882 C}$ overexpressing cells and coincided with sustained p53 activation (Fig. 2D,E). Of note, per-cell levels of a DNA damage marker $\gamma \mathrm{H} 2 \mathrm{~A} . \mathrm{X}$ were also significantly higher (Supplementary Fig. S2D,E) consistent with a defect in DNA damage repair. Mechanistically, this coincided with impaired recruitment of PARP1, a key component in directing DNA repair ${ }^{42}$, to chromatin in $D N M T 3 A^{m u t}$-expressing cells (Fig. 2F).

\section{Cells expressing mutant $D N M T 3 A$ are prone to replication stress and fork collapse}

We hypothesized that increased sensitivity to DNA damage during S-phase and accumulation of unresolved DNA breaks was associated with replication stress. Indeed, this was found in cells expressing mutant DNMT3A as evidenced by high levels of phospho-RPA, which persisted even after drug wash-out (Fig. 3A,B), and coincided with $\gamma \mathrm{H} 2 \mathrm{~A} . \mathrm{X}$ accumulation (Supplementary Fig. S3A,B). Consistently, DNMT3 $A^{\text {mut }}$ expressing cells treated with cytarabine were characterized by a higher number and a larger area of PCNA foci $^{43}$ (Fig. 3C,D). Of note, since Ara-C inhibits elongation, BrdU incorporation, an otherwise ideal means to visualize replication foci, could not be used in these studies.

Next, we examined resumption of DNA synthesis using pulse-chase double-labeling experiments with EdU (to identify replicating cells) and, following cytarabine wash-out, BrdU (marking replication restart). Cells were able to resume replication regardless of the DNMT3A genotype, with similar overall kinetics (Supplementary Fig. S3C,D) despite persisting DNA damage at replication forks. Confocal imaging revealed that $D N M T 3 A$ mutant cells were more likely to suffer fork collapse seen as lack of colocalization between EdU (replication foci pre-cytarabine) and BrdU (replication restart) (Fig. 3E-G).

Cells expressing mutant $D N M T 3 A$ progress through the cell cycle despite persisting DNA damage, leading to mitotic catastrophe 
A

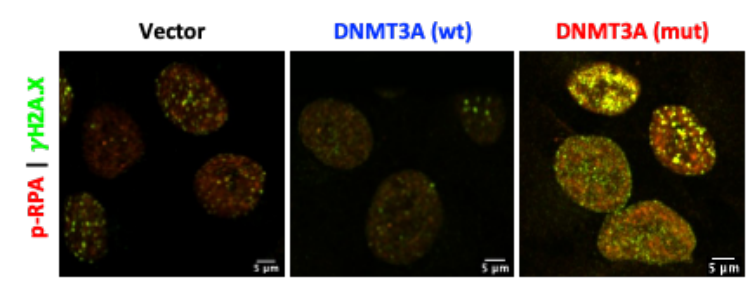

C
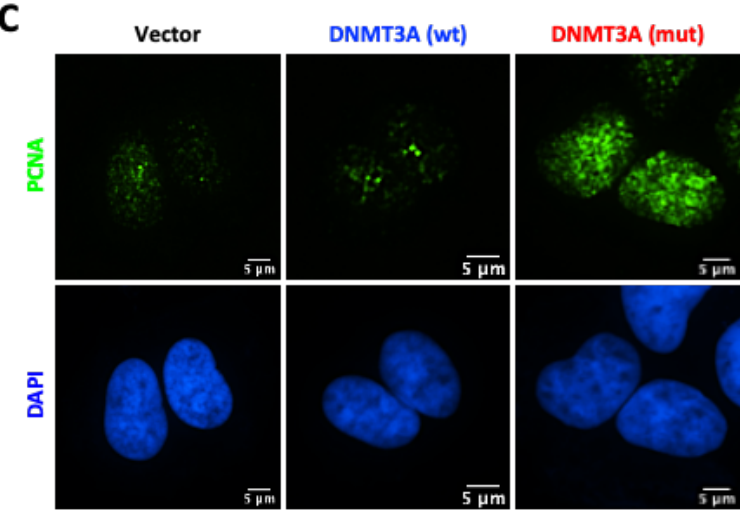

$\mathbf{E}$

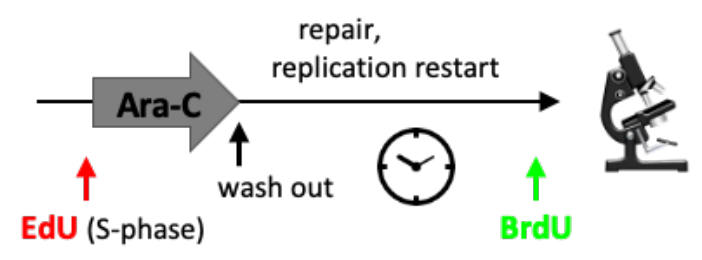

$\mathbf{F}$
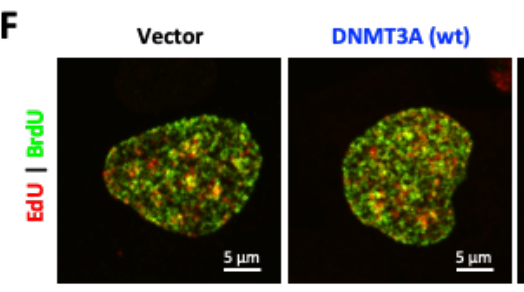

B

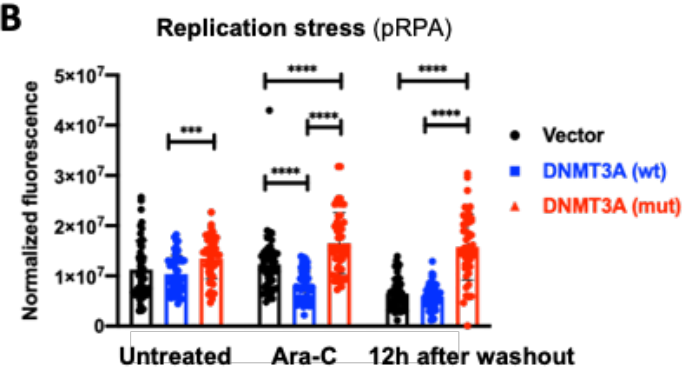

D
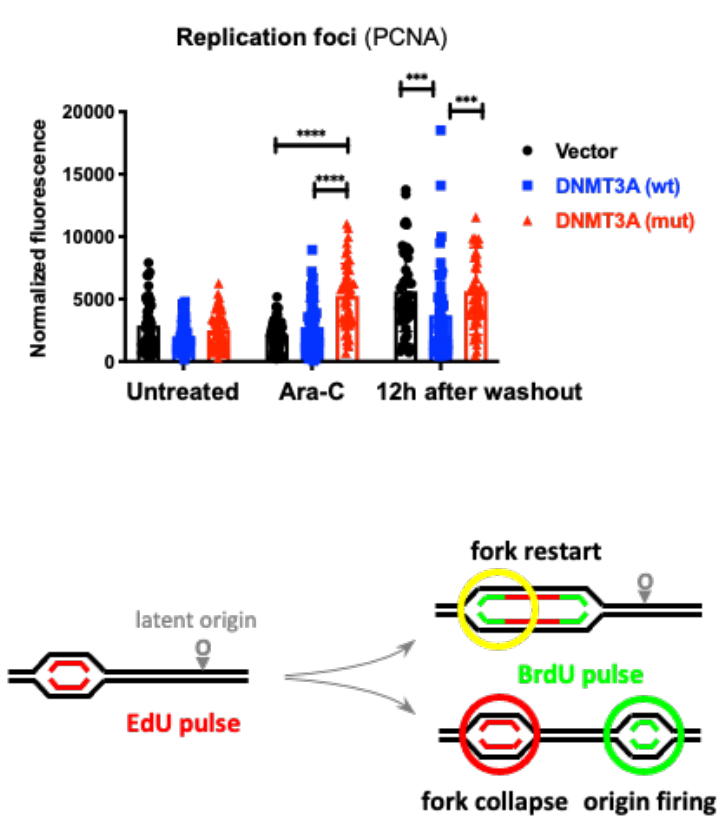

G

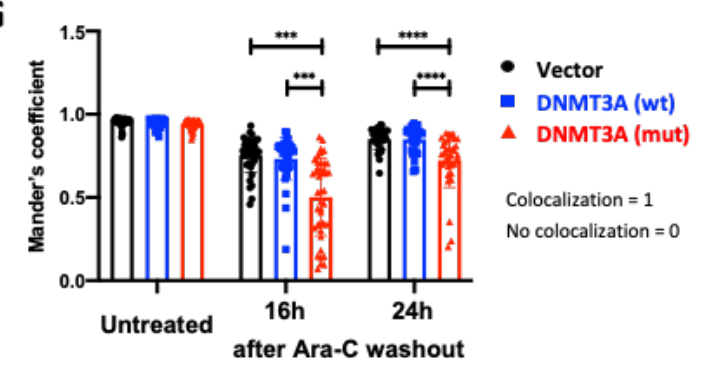

Figure 3. Cells expressing mutant DNMT3A experience prolonged replication stress after cytarabine treatment. (A, B) Replication stress and DNA damage after cytarabine treatment in U2OS cells transduced to express wild-type and mutant DNMT3A or empty vector control. Representative immunofluorescence microscopy images after $12 \mathrm{~h}$ exposure (A, pRPA pSer33, red, and DSB marker $\gamma \mathrm{H} 2 \mathrm{~A} . \mathrm{X}$, green) and pRPA signal quantification at steady state, 12 hours of treatment, and 12 hours after drug wash-out in at least 50 cells per condition (B). (C, D) Analysis of replication foci by PCNA immunofluorescence staining (green) and DAPI (blue) after 12 hours of cytarabine treatment (C) and PCNA signal quantification in untreated, treated, and 12 hours after drug removal in at least 50 cells per condition (D) in U2OS cells with wild-type and mutant DNMT3A. (E-G) Replication restart analysis by EdU and BrdU doublelabeling pulse-chase experiments. U2OS cells overexpressing wild-type and mutant DNMT3A or empty vector control were pulsed with EdU (red) for 1 hour, treated with cytarabine for 12 hours, washed and pulsed with BrdU (green) at 
indicated timepoints (E). Representative immunofluorescence microscopy images of double-labeled cells (F, 16 hours after drug removal) and quantification of BrdU and EdU signal colocalization (Manders coefficient) in at least 25 nuclei per condition at indicated time points after drug removal $(\mathrm{G}) .\left({ }^{* * *} p<0.001, * * * * p<0.0001\right.$, Mann-Whitney rank sum test).

The ability of cytarabine-treated U2OS cells expressing wild-type or mutant DNMT3A to resume cycle progression was determined. The analysis was facilitated by partial cell synchronization at the $\mathrm{G}_{1} / \mathrm{S}_{\text {phase }}$ boundary by Ara-C treatment. By $18 \mathrm{~h}$ after drug wash-out, cells completed replication and began entering mitosis marked by histone $\mathrm{H} 3$ phosphorylation (Fig. 4A and Supplementary Fig. S4A,B). Notably, cells with DNMT3A mutation continued to advance through the cell cycle, albeit with a lag (Supplementary Fig. S4C), despite unresolved DNA damage. As the result, there was an increased frequency of chromosome breaks in metaphase preparations of mutant DNMT3A-expressing cells (Fig. 4B,C) along with hallmarks of mitotic catastrophe such as micronucleation and abnormal nuclear morphology (Fig. 4D-G).

Collectively, our studies indicate that cells with mutant DNMT3A exhibit a defect in repair and recovery of stalled replication forks, resulting in accumulation of DNA breaks carried through mitosis. The accentuated cytarabine sensitivity is further highlighted by our observation that doubling Ara-C concentration prevented mutant DNMT3A-expressing cells from completing replication seen as $\mathrm{G}_{2}$ peak degradation in cell cycle analysis, together with a new sub- $\mathrm{G}_{1}$ population indicative of cell death, in contrast to DNMT3A wild-type cells that maintained a normal cell cycle distribution (Supplementary Fig. S4D).

\section{Gene expression profiling after cytarabine treatment identifies signatures of deregulated cell cycle and DNA replication in cells expressing mutant DNMT3A}

To gain a broader view of cytarabine response, we performed RNA-sequencing to elucidate gene expression signatures deregulated by mutant DNMT3A (Supplementary Table S4). Unsupervised hierarchical clustering of the 2000 most variable genes demonstrated predominant gene activation induced by cytarabine treatment and robust separation from untreated controls, with consistency between biological triplicates (Supplementary Fig. S5A). Pairwise comparisons between cytarabine-treated and untreated control cells 
A

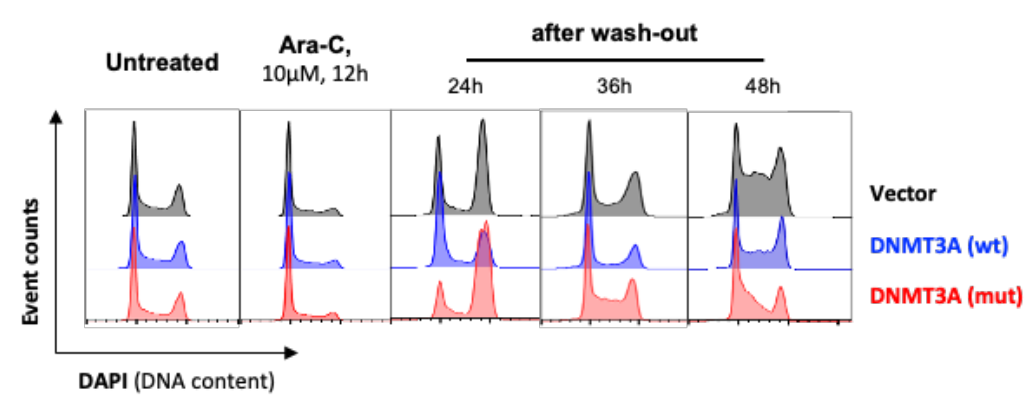

B

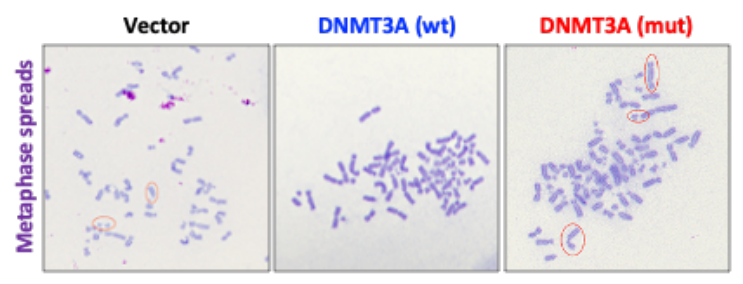

D

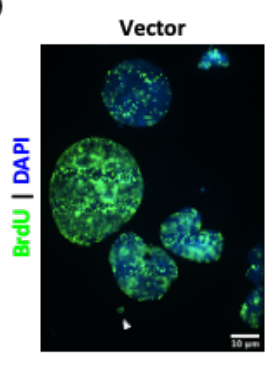

$\mathbf{F}$

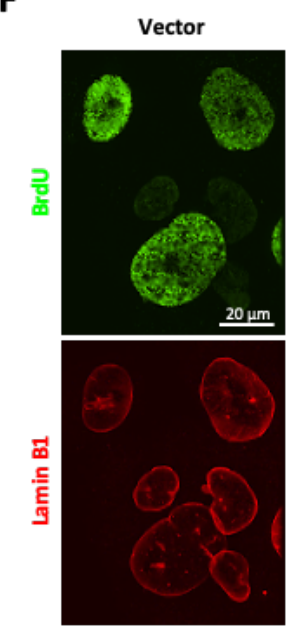

C

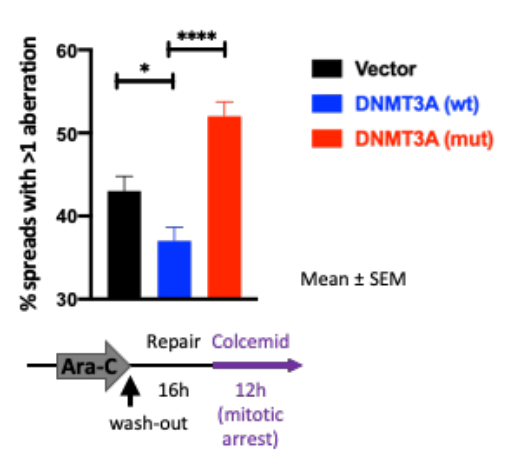

E
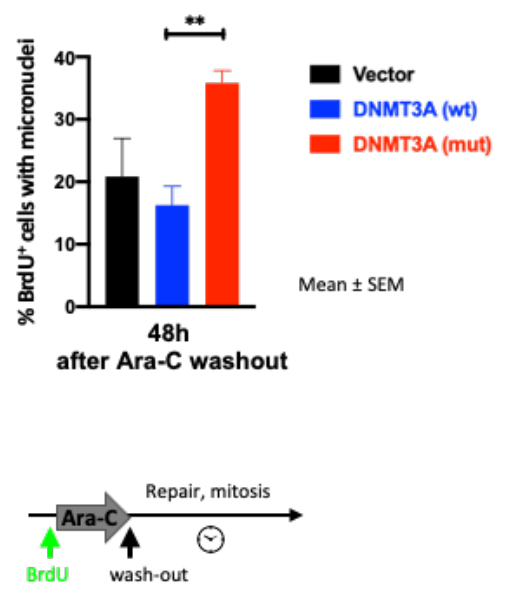

G

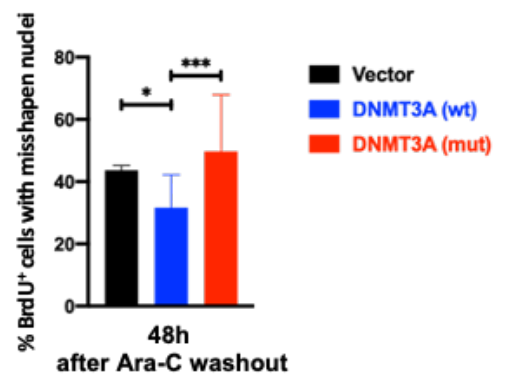

Figure 4. Mutant DNMT3A-expressing cells carrying DNA breaks after cytarabine treatment progress through mitosis. (A) Cell cycle profiles (by DNA content) in U2OS cells with wild-type (blue) and mutant (red) DNMT3A or empty vector control (black) after cytarabine treatment and after wash-out. Fixed and permeabilized cells were stained with DAPI and analyzed by flow cytometry. (B, C) Persistent DNA breaks in mitosis after cytarabine treatment in 
U2OS cells lentivirally overexpressing wild-type and mutant DNMT3A or empty vector control. Representative images of metaphase spreads (B, Wright-Geimsa stain) and frequency of metaphases with chromosomal abnormalities from five independent experiments each scoring at least 200 metaphases per genotype (C) 16 hours after drug washout $(* p<0.05, * * * * p<0.0001$, unpaired $t$-test; graphs represent mean \pm SEM). (D-G) Mitotic catastrophe as a consequence of abnormal mitoses in cells with persistent DNA damage after cytarabine wash-out. Cells were pulsed with $\mathrm{BrdU}$ for $1 \mathrm{~h}$, exposed to cytarabine for $24 \mathrm{~h}$, and released from drug treatment; 48 hours later cells were fixed and stained for BrdU (D, E) and Lamin-B1 (F, G). Representative microphotographs of nuclear morphology (D, DAPI was used to visualize all nuclei in blue) and percentage of $\mathrm{BrdU}^{+}$cells with micronuclei (E, ${ }^{* *} p<0.01$, unpaired $t$ test; graphs represent mean \pm SEM from three independent replicate experiments). Representative microphotographs of nuclear lamina abnormalities visualized by Lamin-B1 staining (red) in $\mathrm{BrdU}^{+}$cells (green) (F) and fraction of $\mathrm{BrdU}^{+}$ cells with abnormally-shaped nuclei (G). At least 100 nuclei per genotype were scored in each of two independent experiments $(* p<0.05, * * * p<0.001$, pair-wise two-tailed Fisher's exact tests, where each normal nucleus was categorized as 0 and each abnormal as 1$)$.

found wide-spread gene activation that was largely shared between genotypes yet featured a significant proportion of uniquely regulated genes in $D N M T 3 A^{m u t}$-expressing samples (Fig. 5A and Supplementary Fig. S5B; Supplementary Table S5).

Pathway analysis using Enrichr to search against HALLMARK gene set collection (MSigDB) and Kyoto Encyclopedia of Genes and Genomes (KEGG) database (Supplementary Table S6) identified p53 signaling as most significantly overrepresented among commonly upregulated genes in all groups (Fig. 5B), consistent with genotoxic stress. At the same time, genes associated with the MYC pathway, G2/M checkpoint, and E2F targets were repressed in all groups, indicative of ongoing replication stress and proliferation delay, reinforcing our biochemical findings (Fig. 2A,E and 4A). Conversely, genes uniquely upregulated in $D N M T 3 A^{m u t}$-expressing cells after cytarabine treatment did not show similarly robust gene set enrichment apart from high expression of genes implicated in stemness and hematopoietic development such as $C D 34^{44}, P D G F R A^{45}, H O X B 6^{46}, I K Z F 2^{47}$ (Supplementary Fig. S5B,C). Analysis of genes with decreased expression after cytarabine uniquely in the mutant DNMT3A-expressing cells highlighted further repression of proliferation-related MYC targets and identified disruption of vital leukemia and stem cell regulatory and metabolic pathways such as mitochondrial oxidative phosphorylation (OXPHOS) and fatty acid metabolism, RNA-splicing, and protein synthesis and degradation ${ }^{48-51}$ (Fig. 5C). Furthermore, functional networks formed by proteins encoded by these exclusively DNMT3A ${ }^{\text {mut }}$-downregulated genes were characterized by prominent clusters annotated as proteasome, mitochondria, spliceosome, and 
bioRxiv preprint doi: https://doi.org/10.1101/2021.05.28.445639; this version posted May 28, 2021. The copyright holder for this preprint (which was not certified by peer review) is the author/funder. All rights reserved. No reuse allowed without permission.

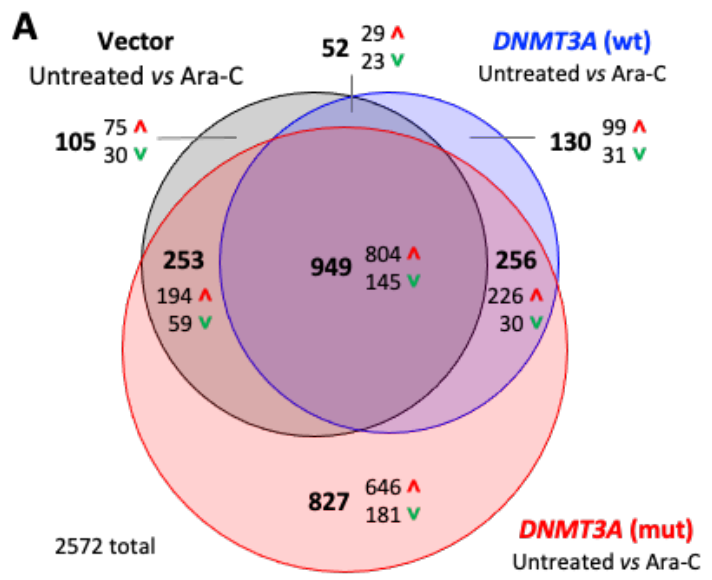

C

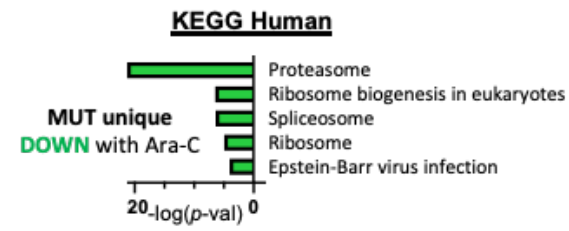

B

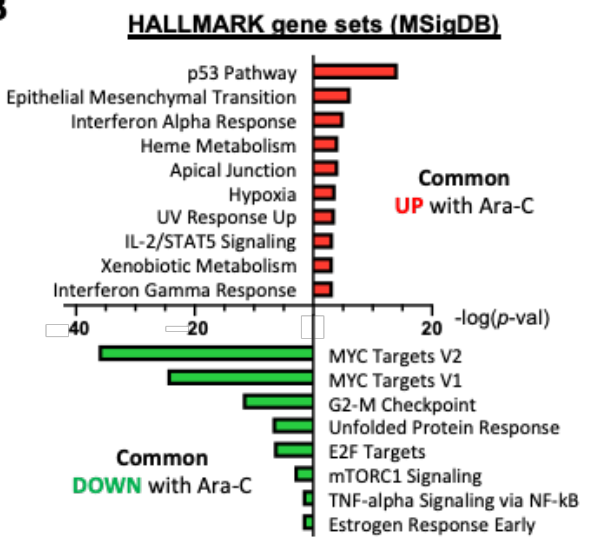

HALLMARK gene sets (MSigDB)

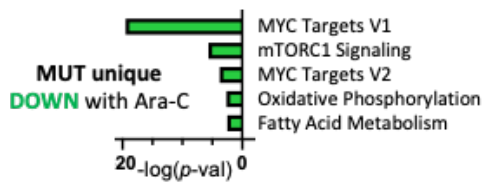

D

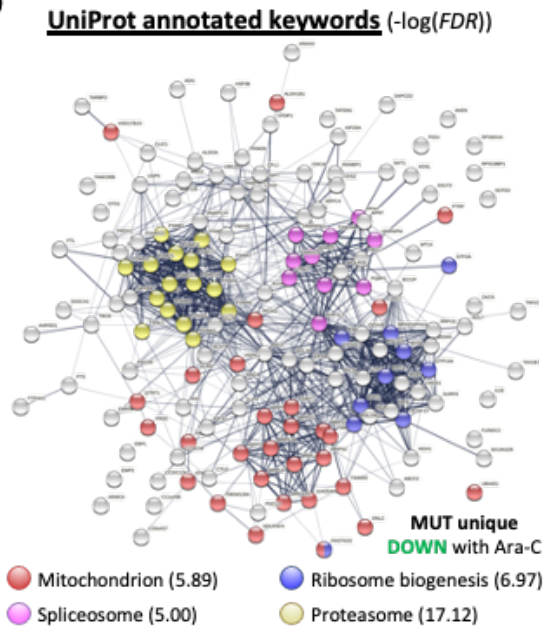

E

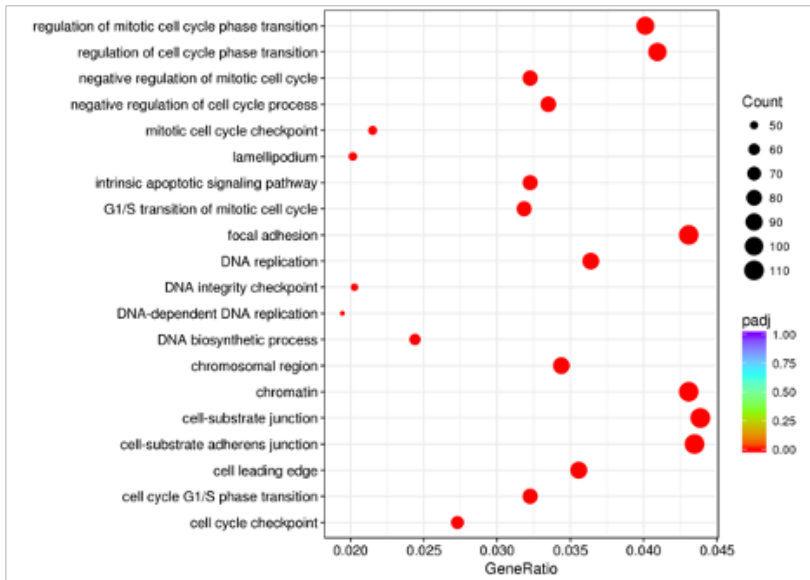

Figure 5. Gene expression profiling identifies pathways deregulated in mutant DNMT3A expressing cells treated with cytarabine. (A) Venn diagram showing differentially expressed genes $(\mathrm{FC} \geq 1.5, \mathrm{FDR}<0.05)$ between vehicle and Ara-C-treated $(10 \mu \mathrm{M}, 24 \mathrm{~h})$ cells that are common or unique to each of the experimental groups: U2OS cells expressing wild-type (blue) or mutant (red) DNMT3A, or empty vector control (black). (B) HALLMARK gene sets (MSigDB) significantly enriched among commonly up- or downregulated genes in all three groups (FDR $<0.1$ ). (C) Significantly enriched KEGG pathways and HALLMARK gene sets among genes uniquely downregulated in $D N M T 3 A^{m u t}$ cells after cytarabine treatment $(\mathrm{FDR}<0.1)$. (D) STRING functional protein network analysis corresponding to genes uniquely downregulated in the $D N M T 3 A^{m u t}$ cells 24 hours after treatment with Ara-C, and their UniProt annotations. (E) Enrichment of GO terms among genes differentially expressed $(p<0.05)$ in cytarabine-treated $D N M T 3 A^{m u t}$ cells compared to treated empty vector controls. 
ribosome biogenesis (Fig. 5D), suggesting distinctive shifts in cell physiology and molecular liabilities.

Pairwise comparison between cytarabine-treated DNMT3A-mutant and empty vector control cells found deregulation of cell cycle-related pathways, emphasizing G1/S phase transition and DNA replication as well as cell adhesion ${ }^{52}$ (Fig. 5E). Gene set enrichment analysis (GSEA) of gene ontology (GO) datasets additionally detected negative enrichment of signatures related to chromatin packaging and myeloid cell differentiation, in line with previous reports and a prominent role of DNMT3A in myeloid biology $5,11,13,24,53$ (Supplementary Fig. S5D).

\section{DISCUSSION}

Mutations in DNMT3A are among the most common genetic alterations in de novo AML and are associated with adverse outcomes ${ }^{6-9,12,14,15,22,54,55}$. In particular, the R882 mutation conveys an unfavorable prognosis in patients $\geq 60$ years of age ${ }^{56}$. This is at least in part due to relative resistance to anthracyclines stemming from another defect in DNA damage response ${ }^{12-14}$ and inability to tolerate dose-sense regimens, straining an already limited list of therapeutic options available for this patient group ${ }^{3,19}$. Here we show that cells with DNMT3A mutations may be sensitive to replication stress-related DNA damage induced by nucleoside analogs such as cytarabine, in agreement with a recent clinical trial ${ }^{21}$. Low-dose cytarabine-, cladribinebased, or other similar regimens are well-tolerated and hence suitable for older patients and those with poor performance status.

A growing body of evidence recognizes emergence of "collateral sensitivities", or increased susceptibility to a second therapy as a fitness trade-off in cancer cells following the development of resistance to initial treatment or other perturbations ${ }^{57-59}$. This raises a possibility of rationally constructing synthetic lethalitybased treatment approaches exploiting such antagonistic pleiotropic mechanisms ${ }^{60,61}$. The concept that the same genetic defect may be protective in the context of a specific type of DNA damage yet make cells vulnerable to a different type of genotoxic insult has been described ${ }^{62}$. Similarly, our studies demonstrate 
whereas the presence of $D N M T 3 A(\mathrm{R} 882)$ decreases the sensitivity to DNA torsional stress induced by anthracyclines and possibly other DNA intercalators ${ }^{13}$, it renders the cells more susceptible to the deleterious effects of replication fork stalling.

Accentuated replication stress in cells expressing mutant DNMT3A is further supported by previous findings of negatively enriched E2F target and CHEK1-regulated $\mathrm{G}_{2} / \mathrm{M}$ checkpoint gene sets reported in independent AML cohorts as well as in mice ${ }^{13,28}$, which reflect $\mathrm{G}_{1} / \mathrm{S}$ cell cycle phase transition rate $\mathrm{e}^{29,30,63}$ and $\mathrm{G}_{2} / \mathrm{M}$ checkpoint adaptation ${ }^{64,65}$. Accordingly, after low-dose cytarabine exposure (approx. $\mathrm{IC}_{20}$ ) mutant DNMT3A-expressing cells demonstrated delayed cell cycle progression that culminated in entering mitosis with damaged DNA. Surviving cells demonstrated increased formation of micronuclei, structures containing shattered chromosomes, indicative of mitotic catastrophe ${ }^{41,66}$. Furthermore, incremental increase in cytarabine concentration resulted in disproportionate apoptotic response, highlighting heightened replication stress in DNMT3A-mutant cells as a potential therapeutic vulnerability that can be unveiled by replication-stalling nucleoside analogs.

Such distinctive sensitivity to cytarabine arises following a defect in recovery from replication fork arrest that leads to accumulation of persistent, unrepaired DNA damage observed in cells with mutant DNMT3A. While previous studies have attributed specific DNA damage repair pathways as defining therapeutic responses to replication stress-inducing nucleoside analogs $\mathrm{s}^{33,34,67-70}$, the exact mechanism(s) that underlie impaired resolution of cytarabine-induced DNA lesions in cells with mutant DNMT3A remain uncertain. Interestingly, other than a slight upregulation of genes annotated to be repressed during UV response indicative of a possible disruption of nucleotide excision repair ${ }^{42}$ (Supplementary Fig. S5C) our gene expression studies did not detect signatures of impaired DNA damage repair. Prior in-depth studies reported only modest changes in DNA methylation ${ }^{13,25-27,71}$ that predominantly affected lineage-specific regulatory elements ${ }^{28,53,72-74}$ yet did not affect DNA damage and repair pathways. Whether a methylation-independent mechanism of mutant DNMT3A action might be at play remains to be determined. In support of this, wildtype DNMT3A protein was found to directly associate with stalled replication forks ${ }^{31}$ and likely enriched 
in the PCNA interactome ${ }^{75}$. There are many examples of non-catalytic accessory functions of epigenetic enzymes regulating both histone $\mathrm{e}^{76,77}$ and DNA modifications ${ }^{78-80}$. Future studies interrogating the replication fork-associated proteome ${ }^{81}$ in cells with and without mutant DNMT3A in addition to differential recruitment of DNA repair proteins to chromatin and proximity-labeling proteomics approaches ${ }^{82,83}$ will be necessary to clarify its role in preserving DNA integrity. This emerging knowledge will be instructive to develop further therapeutic combination strategies.

In addition to DNA replication, cell cycle regulation, and proliferation-related gene sets, which are likely secondary to DNA damage after cytarabine, our gene expression studies uncovered signatures of deregulated cell adhesion, proteostasis, as well as repressed OXPHOS and fatty acid metabolism, and RNA splicing, all of which are critical for hematopoietic stem cell function and are commonly deregulated during aging and in myeloid malignancies. Notably, deregulation of ribosome biogenesis and protein degradation may reflect loss of stemness ${ }^{84,85}$, whereas increased reliance on OXPHOS and fatty acid metabolism are characteristic of chemoresistant acute myeloid leukemia ${ }^{49,52,86}$ and together with altered splicing engender potential therapeutic vulnerabilities ${ }^{50,87,88}$. It will be interesting to investigate if these processes contribute to the leukemic progression in individuals with clonal hematopoiesis with $D N M T 3 A$ mutations ${ }^{89-94}$ to serve as biomarkers for high risk of AML development.

In summary, our studies demonstrate that cells expressing mutant DNMT3A have a defect in recovery from replication fork arrest and subsequent accumulation of unresolved DNA damage, which may have therapeutic tractability. These results demonstrate, in addition to its role in epigenetic control, DNMT3A contributes to preserving genome integrity during DNA replication and suggest that cytarabine-induced replication fork stalling may further synergize with other agents aimed at DNA damage and repair, replication, or cell survival, as well as splicing and oxidative phosphorylation ${ }^{50,51,87,88,95-97}$. Future studies will determine if such combinatorial treatment approaches can be developed to usher in the era of precision oncology for AML patients with mutant DNMT3A. 


\section{ACKNOWLEDGEMENTS}

The authors gratefully acknowledge generous support by NIH awards CA178191 and DK121831 (O.A.G.), CA188561 (R.O.), and CA252400 (B.K.L.). O.A.G. was supported in part by the Ocala Royal Dames for Cancer Research, the Harry T. Mangurian Foundation, and the Thomas H. Maren Junior Investigator Fund. B.K.L. was supported in part by the Florida Breast Cancer Foundation and the Ocala Royal Dames for Cancer Research. J.L. and D.D.R. are Leukemia and Lymphoma Society (LLS) Special Fellows; J.D.L. is supported in part by the LLS Specialized Center of Research (SCOR). Immunophenotyping was performed at the Flow Cytometry and Imaging Core, UF Interdisciplinary Center for Biotechnology Research (ICBR) (RRID: SCR_019119).

\section{AUTHORSHIP CONTRIBUTIONS}

K.V. and O.A.G conceived the study and designed experiments, with conceptual input from J.D.L., R.O., C.R.C., and B.K.L.

K.V. performed experiments, with assistance from Y.F., D.E.S., and O.A.G., and additional help from C.M.B., K.I.K., C.T., Z.Z., H.L.C.R., L.M.P., C.G., J.L., D.D.-R., and R.L.B.

S.K. and P.B.S. performed and analyzed ex vivo drug studies in primary AML samples.

P.N. analyzed RNA-seq dataset, with assistance from A.R. and S.P.

J.E.B. assisted with microscopy experiments and data analysis.

K.V. and O.A.G. analyzed the data and wrote the manuscript, with input from all co-authors.

\section{CONFLICT OF INTEREST DISCLOSURES}

The authors declare no relevant competing financial interests. 


\section{REFERENCES}

1. Dohner H, Weisdorf DJ, Bloomfield CD. Acute Myeloid Leukemia. N Engl J Med. 2015;373(12):11361152.

2. Estey EH. Acute myeloid leukemia: 2019 update on risk-stratification and management. Am J Hematol. 2018;93(10):1267-1291.

3. Tamamyan G, Kadia T, Ravandi F, et al. Frontline treatment of acute myeloid leukemia in adults. Crit Rev Oncol Hematol. 2017;110:20-34.

4. Stein EM, Tallman MS. Emerging therapeutic drugs for AML. Blood. 2016;127(1):71-78.

5. Brunetti L, Gundry MC, Goodell MA. DNMT3A in Leukemia. Cold Spring Harb Perspect Med. 2017;7(2).

6. Cancer Genome Atlas Research N. Genomic and epigenomic landscapes of adult de novo acute myeloid leukemia. N Engl J Med. 2013;368(22):2059-2074.

7. Ley TJ, Ding L, Walter MJ, et al. DNMT3A mutations in acute myeloid leukemia. $N$ Engl J Med. 2010;363(25):2424-2433.

8. Papaemmanuil E, Gerstung M, Bullinger L, et al. Genomic Classification and Prognosis in Acute Myeloid Leukemia. N Engl J Med. 2016;374(23):2209-2221.

9. Yan XJ, Xu J, Gu ZH, et al. Exome sequencing identifies somatic mutations of DNA methyltransferase gene DNMT3A in acute monocytic leukemia. Nat Genet. 2011;43(4):309-315.

10. Yang L, Rau R, Goodell MA. DNMT3A in haematological malignancies. Nat Rev Cancer. 2015;15(3):152-165.

11. Venugopal K, Feng Y, Shabashvili D, Guryanova OA. Alterations to DNMT3A in Hematologic Malignancies. Cancer Res. 2021;81(2):254-263.

12. Patel JP, Gonen M, Figueroa ME, et al. Prognostic relevance of integrated genetic profiling in acute myeloid leukemia. N Engl J Med. 2012;366(12):1079-1089.

13. Guryanova OA, Shank K, Spitzer B, et al. DNMT3A mutations promote anthracycline resistance in acute myeloid leukemia via impaired nucleosome remodeling. Nat Med. 2016;22(12):1488-1495.

14. Sehgal AR, Gimotty PA, Zhao J, et al. DNMT3A Mutational Status Affects the Results of DoseEscalated Induction Therapy in Acute Myelogenous Leukemia. Clin Cancer Res. 2015;21(7):16141620.

15. Balasubramanian SK, Aly M, Nagata Y, et al. Distinct clinical and biological implications of various DNMT3A mutations in myeloid neoplasms. Leukemia. 2018;32(2):550-553.

16. Stone RM, Mandrekar SJ, Sanford BL, et al. Midostaurin plus Chemotherapy for Acute Myeloid Leukemia with a FLT3 Mutation. N Engl J Med. 2017;377(5):454-464. 
17. Lowenberg B, Pabst T, Vellenga E, et al. Cytarabine dose for acute myeloid leukemia. N Engl J Med. 2011;364(11):1027-1036.

18. Burnett AK, Russell N, Hills RK, et al. A randomised comparison of the novel nucleoside analogue sapacitabine with low-dose cytarabine in older patients with acute myeloid leukaemia. Leukemia. 2015;29(6):1312-1319.

19. Sekeres MA, Guyatt G, Abel G, et al. American Society of Hematology 2020 guidelines for treating newly diagnosed acute myeloid leukemia in older adults. Blood Adv. 2020;4(15):3528-3549.

20. Kadia TM, Borthakur G, Pemmaraju N, et al. Phase II Study of Venetoclax Added to Cladribine + Low Dose AraC (LDAC) Alternating with 5-Azacytidine Demonstrates High Rates of Minimal Residual Disease (MRD) Negative Complete Remissions (CR) and Excellent Tolerability in Older Patients with Newly Diagnosed Acute Myeloid Leukemia (AML). Blood. 2020;136(Supplement 1):17-19.

21. Kadia TM, Cortes J, Ravandi F, et al. Cladribine and low-dose cytarabine alternating with decitabine as front-line therapy for elderly patients with acute myeloid leukaemia: a phase 2 single-arm trial. Lancet Haematol. 2018;5(9):e411-e421.

22. Gaidzik VI, Schlenk RF, Paschka P, et al. Clinical impact of DNMT3A mutations in younger adult patients with acute myeloid leukemia: results of the AML Study Group (AMLSG). Blood. 2013;121(23):4769-4777.

23. Ostronoff F, Othus M, Ho PA, et al. Mutations in the DNMT3A exon 23 independently predict poor outcome in older patients with acute myeloid leukemia: a SWOG report. Leukemia. 2013;27(1):238241.

24. Challen GA, Sun D, Jeong M, et al. Dnmt3a is essential for hematopoietic stem cell differentiation. Nat Genet. 2011;44(1):23-31.

25. Russler-Germain DA, Spencer DH, Young MA, et al. The R882H DNMT3A mutation associated with AML dominantly inhibits wild-type DNMT3A by blocking its ability to form active tetramers. Cancer Cell. 2014;25(4):442-454.

26. Cole CB, Russler-Germain DA, Ketkar S, et al. Haploinsufficiency for DNA methyltransferase 3A predisposes hematopoietic cells to myeloid malignancies. J Clin Invest. 2017;127(10):3657-3674.

27. Jeong M, Park HJ, Celik H, et al. Loss of Dnmt3a Immortalizes Hematopoietic Stem Cells In Vivo. Cell Rep. 2018;23(1):1-10.

28. Glass JL, Hassane D, Wouters BJ, et al. Epigenetic Identity in AML Depends on Disruption of Nonpromoter Regulatory Elements and Is Affected by Antagonistic Effects of Mutations in Epigenetic Modifiers. Cancer Discov. 2017;7(8):868-883.

29. Furnari B, Rhind N, Russell P. Cdc25 mitotic inducer targeted by chk1 DNA damage checkpoint kinase. Science. 1997;277(5331):1495-1497. 
30. Branigan TB, Kozono D, Schade AE, et al. MMB-FOXM1-driven premature mitosis is required for CHK1 inhibitor sensitivity. Cell Rep. 2021;34(9):108808.

31. Dungrawala H, Rose KL, Bhat KP, et al. The Replication Checkpoint Prevents Two Types of Fork Collapse without Regulating Replisome Stability. Mol Cell. 2015;59(6):998-1010.

32. Lamba JK. Genetic factors influencing cytarabine therapy. Pharmacogenomics. 2009;10(10):16571674.

33. Ewald B, Sampath D, Plunkett W. Nucleoside analogs: molecular mechanisms signaling cell death. Oncogene. 2008;27(50):6522-6537.

34. Lai TH, Mitchell S, Wu PJ, et al. HSP90 inhibition depletes DNA repair proteins to sensitize acute myelogenous leukemia to nucleoside analog chemotherapeutics. Leuk Lymphoma. 2019:1-4.

35. Vousden KH, Lane DP. p53 in health and disease. Nat Rev Mol Cell Biol. 2007;8(4):275-283.

36. Bieging KT, Mello SS, Attardi LD. Unravelling mechanisms of p53-mediated tumour suppression. Nat Rev Cancer. 2014;14(5):359-370.

37. Berti M, Cortez D, Lopes M. The plasticity of DNA replication forks in response to clinically relevant genotoxic stress. Nat Rev Mol Cell Biol. 2020;21(10):633-651.

38. Sampath D, Rao VA, Plunkett W. Mechanisms of apoptosis induction by nucleoside analogs. Oncogene. 2003;22(56):9063-9074.

39. O'Connor MJ. Targeting the DNA Damage Response in Cancer. Mol Cell. 2015;60(4):547-560.

40. Forment JV, O'Connor MJ. Targeting the replication stress response in cancer. Pharmacol Ther. 2018;188:155-167.

41. Castedo M, Perfettini JL, Roumier T, Andreau K, Medema R, Kroemer G. Cell death by mitotic catastrophe: a molecular definition. Oncogene. 2004;23(16):2825-2837.

42. Ciccia A, Elledge SJ. The DNA damage response: making it safe to play with knives. Mol Cell. 2010;40(2):179-204.

43. Moldovan GL, Pfander B, Jentsch S. PCNA, the maestro of the replication fork. Cell. 2007;129(4):665679.

44. Satterthwaite AB, Burn TC, Le Beau MM, Tenen DG. Structure of the gene encoding CD34, a human hematopoietic stem cell antigen. Genomics. 1992;12(4):788-794.

45. Demoulin JB, Montano-Almendras CP. Platelet-derived growth factors and their receptors in normal and malignant hematopoiesis. Am J Blood Res. 2012;2(1):44-56.

46. Alharbi RA, Pettengell R, Pandha HS, Morgan R. The role of HOX genes in normal hematopoiesis and acute leukemia. Leukemia. 2013;27(5):1000-1008.

47. Park SM, Cho H, Thornton AM, et al. IKZF2 Drives Leukemia Stem Cell Self-Renewal and Inhibits Myeloid Differentiation. Cell Stem Cell. 2019;24(1):153-165 e157. 
48. Signer RA, Magee JA, Salic A, Morrison SJ. Haematopoietic stem cells require a highly regulated protein synthesis rate. Nature. 2014;509(7498):49-54.

49. Farge T, Saland E, de Toni F, et al. Chemotherapy-Resistant Human Acute Myeloid Leukemia Cells Are Not Enriched for Leukemic Stem Cells but Require Oxidative Metabolism. Cancer Discov. 2017;7(7):716-735.

50. Crews LA, Balaian L, Delos Santos NP, et al. RNA Splicing Modulation Selectively Impairs Leukemia Stem Cell Maintenance in Secondary Human AML. Cell Stem Cell. 2016;19(5):599-612.

51. Faubert B, Solmonson A, DeBerardinis RJ. Metabolic reprogramming and cancer progression. Science. 2020;368(6487).

52. Raffel S, Klimmeck D, Falcone M, et al. Quantitative proteomics reveals specific metabolic features of acute myeloid leukemia stem cells. Blood. 2020;136(13):1507-1519.

53. Guryanova OA, Lieu YK, Garrett-Bakelman FE, et al. Dnmt3a regulates myeloproliferation and liverspecific expansion of hematopoietic stem and progenitor cells. Leukemia. 2016;30(5):1133-1142.

54. Ribeiro AF, Pratcorona M, Erpelinck-Verschueren C, et al. Mutant DNMT3A: a marker of poor prognosis in acute myeloid leukemia. Blood. 2012;119(24):5824-5831.

55. Metzeler KH, Herold T, Rothenberg-Thurley M, et al. Spectrum and prognostic relevance of driver gene mutations in acute myeloid leukemia. Blood. 2016;128(5):686-698.

56. Marcucci G, Metzeler KH, Schwind S, et al. Age-related prognostic impact of different types of DNMT3A mutations in adults with primary cytogenetically normal acute myeloid leukemia. $J$ Clin Oncol. 2012;30(7):742-750.

57. Singleton KR, Crawford L, Tsui E, et al. Melanoma Therapeutic Strategies that Select against Resistance by Exploiting MYC-Driven Evolutionary Convergence. Cell Rep. 2017;21(10):2796-2812.

58. Zhao B, Sedlak JC, Srinivas R, et al. Exploiting Temporal Collateral Sensitivity in Tumor Clonal Evolution. Cell. 2016;165(1):234-246.

59. Wang L, Leite de Oliveira R, Huijberts S, et al. An Acquired Vulnerability of Drug-Resistant Melanoma with Therapeutic Potential. Cell. 2018;173(6):1413-1425 e1414.

60. Lin KH, Rutter JC, Xie A, et al. Using antagonistic pleiotropy to design a chemotherapy-induced evolutionary trap to target drug resistance in cancer. Nat Genet. 2020;52(4):408-417.

61. Acar A, Nichol D, Fernandez-Mateos J, et al. Exploiting evolutionary steering to induce collateral drug sensitivity in cancer. Nat Commun. 2020;11(1):1923.

62. Eccles LJ, Bell AC, Powell SN. Inhibition of non-homologous end joining in Fanconi Anemia cells results in rescue of survival after interstrand crosslinks but sensitization to replication associated double-strand breaks. DNA Repair (Amst). 2018;64:1-9.

63. Elledge SJ. Cell cycle checkpoints: preventing an identity crisis. Science. 1996;274(5293):1664-1672. 
64. Lewis CW, Golsteyn RM. Cancer cells that survive checkpoint adaptation contain micronuclei that harbor damaged DNA. Cell Cycle. 2016;15(22):3131-3145.

65. Kalsbeek D, Golsteyn RM. G2/M-Phase Checkpoint Adaptation and Micronuclei Formation as Mechanisms That Contribute to Genomic Instability in Human Cells. Int J Mol Sci. 2017;18(11).

66. Imreh G, Norberg HV, Imreh S, Zhivotovsky B. Chromosomal breaks during mitotic catastrophe trigger gammaH2AX-ATM-p53-mediated apoptosis. J Cell Sci. 2016;129(9):1950.

67. Takahashi M, Shimodaira H, Andreutti-Zaugg C, Iggo R, Kolodner RD, Ishioka C. Functional analysis of human MLH1 variants using yeast and in vitro mismatch repair assays. Cancer Res. 2007;67(10):4595-4604.

68. Strom SS, Estey E, Outschoorn UM, Garcia-Manero G. Acute myeloid leukemia outcome: role of nucleotide excision repair polymorphisms in intermediate risk patients. Leuk Lymphoma. 2010;51(4):598-605.

69. Fordham SE, Matheson EC, Scott K, Irving JA, Allan JM. DNA mismatch repair status affects cellular response to Ara-C and other anti-leukemic nucleoside analogs. Leukemia. 2011;25(6):1046-1049.

70. Boeckemeier L, Kraehenbuehl R, Keszthelyi A, et al. Mre11 exonuclease activity removes the chainterminating nucleoside analog gemcitabine from the nascent strand during DNA replication. Sci Adv. 2020;6(22):eaaz4126.

71. Spencer DH, Russler-Germain DA, Ketkar S, et al. CpG Island Hypermethylation Mediated by DNMT3A Is a Consequence of AML Progression. Cell. 2017;168(5):801-816 e813.

72. Izzo F, Lee SC, Poran A, et al. DNA methylation disruption reshapes the hematopoietic differentiation landscape. Nat Genet. 2020;52(4):378-387.

73. Tovy A, Reyes JM, Gundry MC, et al. Tissue-Biased Expansion of DNMT3A-Mutant Clones in a Mosaic Individual Is Associated with Conserved Epigenetic Erosion. Cell Stem Cell. 2020;27(2):326335 e324.

74. Lim JY, Duttke SH, Baker TS, et al. DNMT3A haploinsufficiency causes dichotomous DNA methylation defects at enhancers in mature human immune cells. J Exp Med. 2021;218(7).

75. Srivastava M, Chen Z, Zhang H, et al. Replisome Dynamics and Their Functional Relevance upon DNA Damage through the PCNA Interactome. Cell Rep. 2018;25(13):3869-3883 e3864.

76. Dorighi KM, Swigut T, Henriques T, et al. M113 and M114 Facilitate Enhancer RNA Synthesis and Transcription from Promoters Independently of H3K4 Monomethylation. Mol Cell. 2017;66(4):568576 e564.

77. Wang SP, Tang Z, Chen CW, et al. A UTX-MLL4-p300 Transcriptional Regulatory Network Coordinately Shapes Active Enhancer Landscapes for Eliciting Transcription. Mol Cell. 2017;67(2):308-321 e306. 
78. Palii SS, Van Emburgh BO, Sankpal UT, Brown KD, Robertson KD. DNA methylation inhibitor 5Aza-2'-deoxycytidine induces reversible genome-wide DNA damage that is distinctly influenced by DNA methyltransferases 1 and 3B. Mol Cell Biol. 2008;28(2):752-771.

79. Nowialis P, Lopusna K, Opavska J, et al. Catalytically inactive Dnmt3b rescues mouse embryonic development by accessory and repressive functions. Nat Commun. 2019;10(1):4374.

80. Lu R, Wang P, Parton T, et al. Epigenetic Perturbations by Arg882-Mutated DNMT3A Potentiate Aberrant Stem Cell Gene-Expression Program and Acute Leukemia Development. Cancer Cell. 2016;30(1):92-107.

81. Sirbu BM, Couch FB, Cortez D. Monitoring the spatiotemporal dynamics of proteins at replication forks and in assembled chromatin using isolation of proteins on nascent DNA. Nat Protoc. 2012;7(3):594-605.

82. Chen CL, Perrimon N. Proximity-dependent labeling methods for proteomic profiling in living cells. Wiley Interdiscip Rev Dev Biol. 2017;6(4).

83. Bosch JA, Chen CL, Perrimon N. Proximity-dependent labeling methods for proteomic profiling in living cells: An update. Wiley Interdiscip Rev Dev Biol. 2021;10(1):e392.

84. Hidalgo San Jose L, Sunshine MJ, Dillingham CH, et al. Modest Declines in Proteome Quality Impair Hematopoietic Stem Cell Self-Renewal. Cell Rep. 2020;30(1):69-80 e66.

85. Chavez JS, Rabe JL, Loeffler D, et al. PU.1 enforces quiescence and limits hematopoietic stem cell expansion during inflammatory stress. $J$ Exp Med. 2021;218(6).

86. Tcheng M, Roma A, Ahmed N, et al. Very long chain fatty acid metabolism is required in acute myeloid leukemia. Blood. 2021.

87. Molina JR, Sun Y, Protopopova M, et al. An inhibitor of oxidative phosphorylation exploits cancer vulnerability. Nat Med. 2018;24(7):1036-1046.

88. Escobar-Hoyos L, Knorr K, Abdel-Wahab O. Aberrant RNA Splicing in Cancer. Annu Rev Cancer Biol. 2019;3(1):167-185.

89. Abelson S, Collord G, Ng SWK, et al. Prediction of acute myeloid leukaemia risk in healthy individuals. Nature. 2018;559(7714):400-404.

90. Corces-Zimmerman MR, Hong WJ, Weissman IL, Medeiros BC, Majeti R. Preleukemic mutations in human acute myeloid leukemia affect epigenetic regulators and persist in remission. Proc Natl Acad Sci U S A. 2014;111(7):2548-2553.

91. Desai P, Mencia-Trinchant N, Savenkov O, et al. Somatic mutations precede acute myeloid leukemia years before diagnosis. Nat Med. 2018;24(7):1015-1023.

92. Jaiswal S, Fontanillas P, Flannick J, et al. Age-related clonal hematopoiesis associated with adverse outcomes. N Engl J Med. 2014;371(26):2488-2498. 
93. Shlush LI, Mitchell A, Heisler L, et al. Tracing the origins of relapse in acute myeloid leukaemia to stem cells. Nature. 2017;547(7661):104-108.

94. Xie M, Lu C, Wang J, et al. Age-related mutations associated with clonal hematopoietic expansion and malignancies. Nat Med. 2014;20(12):1472-1478.

95. Wei AH, Strickland SA, Jr., Hou JZ, et al. Venetoclax Combined With Low-Dose Cytarabine for Previously Untreated Patients With Acute Myeloid Leukemia: Results From a Phase Ib/II Study. J Clin Oncol. 2019;37(15):1277-1284.

96. Webster JA, Tibes R, Morris L, et al. Randomized phase II trial of cytosine arabinoside with and without the CHK1 inhibitor MK-8776 in relapsed and refractory acute myeloid leukemia. Leuk Res. 2017;61:108-116.

97. Leon TE, Rapoz-D'Silva T, Bertoli C, et al. EZH2-Deficient T-cell Acute Lymphoblastic Leukemia Is Sensitized to CHK1 Inhibition through Enhanced Replication Stress. Cancer Discov. 2020;10(7):9981017. 


\section{SUPPLEMENTARY INFORMATION}

\section{DNMT3A harboring leukemia-associated mutations directs sensitivity to DNA damage at replication forks} Venugopal et al.

This document includes supplementary methods, supplementary figures 1-5, supplementary references, and supplementary tables 1-3 (supplementary tables 4-6 are available online).

Supplementary Figure S1. DNMT3A mutations confer sensitivity to replication stalling drugs.

Supplementary Figure S2. Cells expressing mutant DNMT3A accumulate DNA damage due to incomplete DNA repair after cytarabine

Supplementary Figure S3. Cells expressing mutant DNMT3A accumulate DNA damage after cytarabine treatment yet are able to restart replication.

Supplementary Figure S4. Delayed cell cycle progression in the cells expressing mutant DNMT3A after cytarabine treatment.

Supplementary Figure S5. Gene expression profiling identifies pathways deregulated in cells expressing mutant DNMT3A after cytarabine treatment.

Supplementary Table S1. Primary antibodies used for immunoblotting analyses.

Supplementary Table S2. Primary antibodies used for intracellular flow cytometry.

Supplementary Table S3. Primary antibodies used for immunofluorescence.

Supplementary Table S4. (online) RNA-seq: filtered (-10) and normalized gene counts (TMM).

Supplementary Table S5. (online) Differentially expressed genes (untreated vs Ara-C) and overlap between groups

Supplementary Table S6. (online) Pathway analysis of genes commonly or uniquely differentially regulated between groups after cytarabine treatment. 


\section{Supplementary methods}

\section{Animal studies}

A conditional Dnmt $3 a^{R 878 H}$ knock-in mouse line (Jackson Laboratory stock No. 031514) was previously described ${ }^{1}$. Expression of the mutant form of $D n m t 3 a$, also referred to as $D n m t 3 a^{m u t}$, is activated by a hematopoietic-specific $M x 1$-Cre deletor following poly(I:C) injections; poly(I:C)-treated $D n m t 3 a^{+/+}: M x 1-\mathrm{Cre}^{+}$are used as controls. Successful recombination and expression of the point mutant is verified by Sanger sequencing of the peripheral blood nucleated cell cDNA as previously reported.

To generate mouse leukemias with and without Dnmt $3 a^{R 878 H}$, we intercrossed Dnmt $3 a^{R 878 H}:$ Mxl-Cre mice with animals harboring constitutive $F l t 3^{I T D}$ and inducible $N p m 1^{c}$ alleles as previously described. Bone marrow from $D n m t 3 a^{m u t}: F l t 3^{I T D}: N p m I^{c}$ and control Dnmt $3 a^{w t}: F l t 3^{I T D}: N p m I^{c}$ animals developing leukemia after Cre induction (WBC reach or exceed $50 \times 10^{3} / \mu \mathrm{l}$ ) is harvested for ex vivo drug dose response analyses.

For in vivo cytarabine treatment studies, $0.5 \times 10^{6} \mathrm{CD} 45.2$ donor bone marrow cells from fully excised

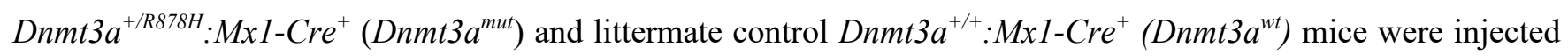
through tail veins into lethally irradiated congenic CD45.1 recipients and allowed to engraft for 6 weeks. Successfully transplanted animals of both genotypes, confirmed by CD45.1/CD45.2 peripheral blood chimerism, were randomized between treatment ( $30 \mathrm{mg} / \mathrm{kg}$ cytarabine/day for 5 days IP) and vehicle (PBS) ( $n=5 /$ group). Bone marrow was harvested $48 \mathrm{~h}$ after last injection by spin-flush method and red blood cell lysis. Analysis of the bone marrow cellular composition was done by flow cytometry. Apoptosis in the stem/progenitor-enriched Lineage ${ }^{-} \mathrm{Scal}^{+} \mathrm{cKit}^{+}$(LSK) population was determined by Annexin V (556422, BD Pharmingen) binding and 4',6diamidino-2-phenylindole (DAPI) uptake (Thermo Fisher). All cell surface antibodies were from eBioscience or BioLegend: NK1.1 (PK136), CD11b (M1/70), CD45R (RA3-6B2), CD3 (17A2), Gr-1 (RB6-8C5), Ter119 (TER119), CD19 (6D5), CD4 (GK1.5), CD8 (53-6.7), cKit (2B8), Sca-1 (D7), CD150 (TC15-12F12.2), CD48 (HM48-1), CD16/32 (93), CD34 (RAM34), Ki67 (16A8), CD45.1 (A20), CD45.2 (104).

\section{Primary AML samples}

Bone marrow aspirates from patients with AML driven by $F L T 3^{I T D}$ and $N P M 1^{c}$, with $(n=4)$ and without $(n=5)$ $D N M T 3 A^{R 882}$, were subjected to ex vivo drug dose response study as previously described ${ }^{1}$, approved by the ethical committee of the Medical University of Vienna \#EK Nr: 2008/2015.

\section{Apoptosis assay}


Apoptosis was measured by flow cytometry using Annexin V (APC) and propidium iodide (PI) double staining. Cells were treated as indicated, collected, counted, and $1 \times 10^{6}$ cells were washed with ice-cold PBS twice, and resuspended in $200 \mu \mathrm{l}$ of $1 \mathrm{X}$ binding buffer containing Annexin V and PI according to the manufacturer's instructions for 15 minutes at $37^{\circ} \mathrm{C}$ in the dark. The proportion of viable, apoptotic and necrotic cells was quantified by flow cytometry and analyzed by the FlowJo software.

\section{Cell growth assay by methylene blue staining}

Lentivirally transduced U2OS cells were plated $1 \times 10^{3}$ cells/well in 96-well plates. Every day, triplicate wells were fixed and stained by $0.5 \%$ methylene blue (Sigma) in 50\% methanol, then washed in water and allowed to air-dry. At experiment completion, bound methylene blue was solubilized in $0.1 \%$ SDS, and $\mathrm{OD}_{595}$ was measured using the SpectraMax plate reader. The amount of bound methylene blue is proportional to the cell number; relative cell growth was normalized to day 0 set at 1 .

\section{Immunoblotting}

Cells treated with Ara-C were collected after washing in ice-cold PBS and lysed in RIPA buffer supplemented with protease and phosphatase inhibitors. Protein concentrations were determined using BCA protein assay (Pierce). For target detection by Western blotting, $25 \mu \mathrm{g}$ of total protein were resolved by SDS-PAGE on 4-12\% Bis-Tris gels (Invitrogen), blotted onto PVDF membranes (Millipore) and probed by standard methods. Primary antigen-specific antibodies, listed in Supplementary Table S1, were used at 1:1000 dilutions and signals were detected after incubation with secondary HRP-linked species-specific antibodies (Santa Cruz Biotech, 1:5000 dilution) using KwikQuant chemiluminescence reagents and a digital imaging station (Kindle Biosciences).

\section{Comet Assay}

Alkaline comet assays were performed using CometSlides and Comet Assay kit (Trevigen), according to manufacturer's instructions. Cells were treated as indicated, harvested, counted, and embedded in low melting point agarose buffer layered onto CometSlides. After processing and electrophoresis, slides were stained with SYBR Gold and imaged using EVOS FL (Life Technologies) imaging system using a 10× NA 0.30 objective. Images were analyzed using ImageJ software (NIH) and an OpenComet plugin calculating percent damaged DNA on the basis of comet head and tail sizes (measured in pixels) and their integral fluorescence intensity. 


\section{Cell cycle analysis}

Cell cycle was analyzed based on DNA content by DAPI staining. Cells were treated as indicated, harvested, and counted. Adherent cells were trypsinized as necessary. One million cells were washed in PBS and fixed in 3.7\% formaldehyde for 15 minutes at room temperature (RT) and permeabilized in 0.5\% Triton X-100 in PBS for 15 minutes. Alternatively, cells were extensively washed and fixed in $70 \%$ ice-cold ethanol at $4{ }^{\circ} \mathrm{C}$ overnight. The cells were then washed in PBS, stained with $1 \mu \mathrm{g} / \mathrm{ml}$ DAPI (Thermo Fisher Scientific) in PBS for 10 minutes, and analyzed by flow cytometry using BD LSR Fortessa. Where indicated, cells were incubated with antibodies against phosphorylated histone H3 (pS28) or H2A.X (pS139) conjugated to AlexaFluor647, for 20 minutes. After washing with PBS, DNA was counterstained using $1 \mu \mathrm{g} / \mathrm{ml}$ DAPI (Thermo Fisher) in PBS for 10 minutes at RT, and analyzed by flow cytometry. DNA content histograms were generated using FlowJo software version 10.4.1, using the CellCycle plugin as appropriate.

\section{BrdU and EdU pulse-chase double-labeling experiments}

U2OS cells were pulsed with EdU $(10 \mu \mathrm{M})(\mathrm{C} 10340$, Invitrogen) for 30 minutes. The cells were then washed and treated with $10 \mu \mathrm{M}$ of Ara-C for 12 hours and then released in $\mathrm{BrdU}(10 \mu \mathrm{M})$ for 30 minutes at the indicated time points. Cells were fixed and permeabilized as described above. Cells were incubated in $2 \mathrm{M} \mathrm{HCl}$ solution for 30 minutes at RT and neutralized in a solution containing 3\% BSA in PBS. EdU was detected using the Click-iT EdU AlexaFluor647 imaging kit (C10340, Invitrogen) according to the manufacturer's protocol. Cells were then incubated in blocking buffer $(0.1 \%$ BSA, $0.05 \%$ Tween 20, 0.2\% TritonX-100, 10\% donkey serum) for 1 hour at RT followed by 1:200 mouse anti-BrdU monoclonal antibody in blocking buffer overnight at $4^{\circ} \mathrm{C}$. After washing in PBS, cells were incubated with AlexaFluor488-labelled donkey anti-mouse secondary antibody (Invitrogen) (1: 1000) in blocking buffer for 1 hour at RT and washed twice in PBS. Coverslips were mounted with ProLong Gold with DAPI (Invitrogen). Slides were examined on Nikon Eclipse Ti2 confocal microscope using a $63 \times$ NA 1.46 objective. For quantitative analyses, at least 50 nuclei were selected at random and analyzed using the ImageJ or NiS software. Colocalization was quantified using Manders overlap coefficient, which measures the degree of overlap of two separate fluorescence channels relative to the total intensity within each channel. A Manders coefficient of 0 corresponds to no colocalization, whereas a value of 1 means $100 \%$ colocalization.

\section{Analysis of micronucleation and nuclear morphology}

Exponentially growing U2OS cells on glass coverslips were pulsed with $\mathrm{BrdU}(10 \mu \mathrm{M})$ for 60 minutes, washed with PBS, treated with $10 \mu \mathrm{M}$ Ara-C for 24 hours, then released into complete media for the indicated duration of time. 
Cells were fixed, permeabilized, and extracted in $2 \mathrm{M} \mathrm{HCl}$ as discussed above. Coverslips were then incubated in blocking buffer followed by anti-BrdU mouse monoclonal antibody and anti-Lamin B1 rabbit polyclonal antibody (ab16048, Abcam) in blocking buffer overnight at $4^{\circ} \mathrm{C}$. After washing in PBS, cells were incubated with AlexaFluor488-labelled or AlexaFluor594-conjugated donkey anti-mouse or anti-rabbit secondary antibodies, washed twice in PBS, and mounted in ProLong Gold mounting medium with DAPI. The images were taken on the Nikon Eclipse Ti2 confocal microscope using a 100× NA 1.49 objective. At least 100 randomly selected BrdUpositive nuclei were counted for presence of micronuclei, or analyzed for eccentricity using the ImageJ and the NiS Elements software.

\section{RNA-seq analysis}

RNA integrity and purity were assessed by Qubit and Agilent 2100 Bioanalyzer (Agilent Technologies, Santa Clara, CA), and high-quality samples with RIN $>8.0$ were sent to Novogene Co. Ltd for library preparation and sequencing. NEBNext ${ }^{\circledR}$ Ultra $^{\text {TM }}$ RNA Library Prep Kit was used for poly-A tailed mRNA enrichment, fragmentation, cDNA synthesis, NEBNext hairpin loop adaptor addition and library preparation. The library quality and insert size were assessed on Agilent 2100 Bioanalyzer and the concentration was determined by Qubit and qPCR methods. The libraries were then sequenced on an Illumina NovaSeq 6000 to generate PE 150 reads.

Raw reads that were pre-processed to remove low quality reads, poly-Ns and adapter sequences were provided in FASTQ format by Novogene and used for all downstream analyses. Clean FASTQ reads were uploaded into PartekFlow ${ }^{\circledR}$ software, version 8.0 (8.0.19.0322 build, Partek, Inc.) on a standalone server at UF ICBR, followed by pre-alignment QA/QC. These reads were then mapped to the human reference genome build 38 (hg38) with the default settings in the STAR aligner version 2.5.3a followed by post-alignment QA/QC showing average alignment rate $93-95 \%$ and 56.4 million transcriptome-aligned reads per sample. The transcript read count was obtained using the default strict paired end compatibility and minimum reads of 10 in the Quantify to Annotation Model (Partek E/M) method and quantified to human hg38 - Ensembl Transcripts release 95. About 80\% of the reads mapped fully to the exons indicating an overall good read distribution and rRNA depletion. Low expression genes (read counts $<10$ ) were filtered to reduce noise and raw read counts across samples were normalized using TMM with an offset of 1 (to avoid zero counts during further logarithmic transformation).

For differential gene expression analysis, first unsupervised hierarchical clustering was performed on $\log 2$ transformed TMM values using Pearson correlation clustering with average linkage. Cluster $3.0^{2}$ was used to $\log 2$ transform input values, filter top 2000 most variable genes and cluster the data. Morpheus online tool (https://software.broadinstitute.org/morpheus) was used for visualization. Heatmap used color coding where minimum and maximum values were set for each row individually. Differentially expressed genes between vehicle 
and cytarabine-treated cells in each genotype were determined by edgeR quasi-likelihood (QL) pipeline ${ }^{3}$ and filtered based on fold change $\geq 1.5$ and FDR $<0.05$. Overlap between groups and proportional Venn diagrams were constructed by Venny online javascript tool (https://github.com/benfred/venn.js; https://www.stefanjol.nl/venny). Gene ontology (GO) and pathway enrichment analyses were done using Enrichr ${ }^{4,5}$ and GSEA ${ }^{6,7}$ to search against MSigDB Hallmark ${ }^{8}$ and $\mathrm{KEGG}^{9}$ databases; enrichment of protein-protein association networks was visualized using STRING ${ }^{10-12}$ application with default parameters. 
A

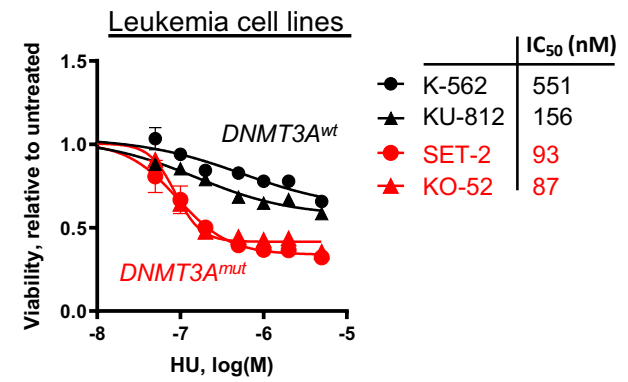

C

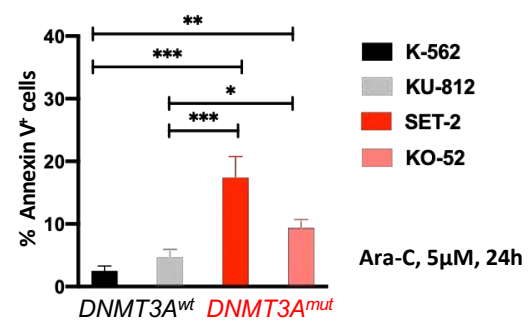

B

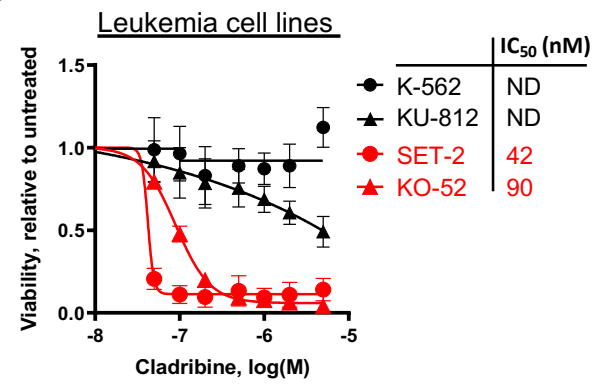

D

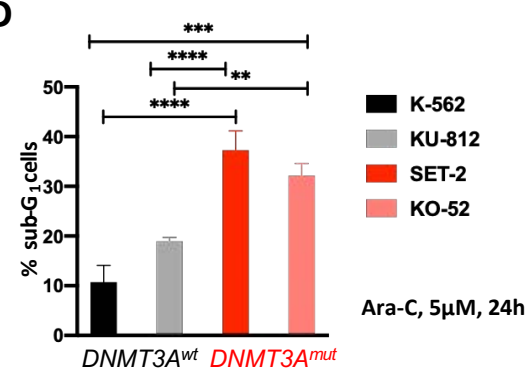

$\mathbf{E}$

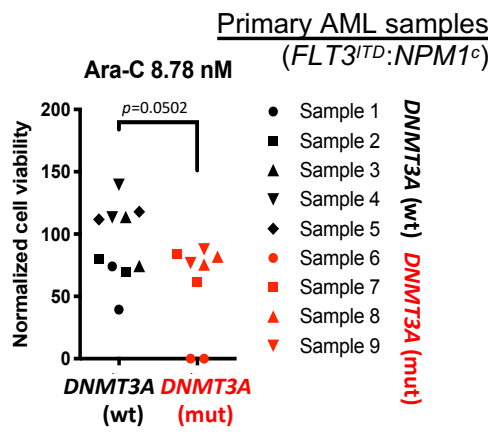

$\mathbf{F}$

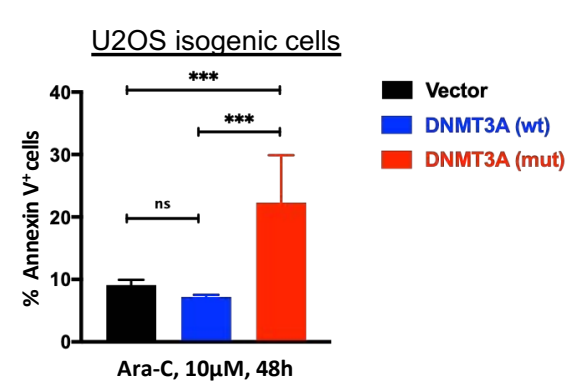

G

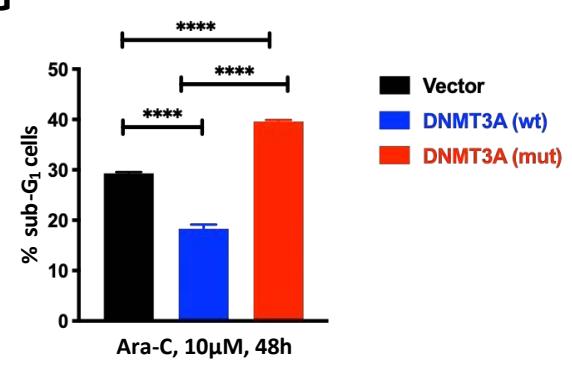

H

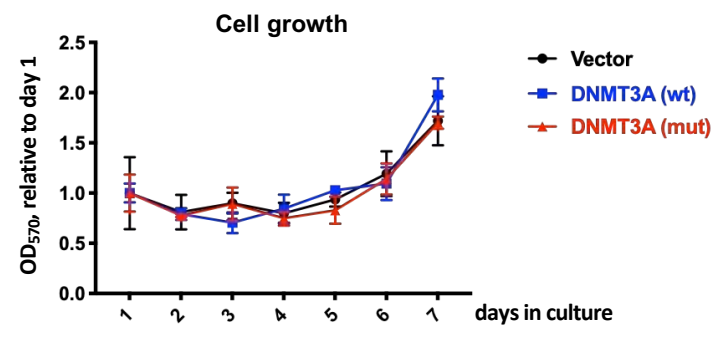




\section{Supplementary Figure S1. DNMT3A mutations confer sensitivity to replication stalling drugs.}

(A, B) Leukemia cells with DNMT3A mutations are more sensitive to replication stalling agents. Drug dose response in leukemia cells lines with wild-type DNMT3A (K-562 and KU-812, black) or with a DNMT3A R882 mutation (SET2 and KO-52, red), exposed to increasing concentrations of hydroxyurea (HU, A) and cladribine (B) for 48 hours. Cell viability was measured by CellTiter GLO assay, in triplicate and normalized to vehicle-treated controls.

(C, D) Apoptosis in leukemia cell lines with wild-type or mutant DNMT3A, treated with $5 \mu \mathrm{M}$ cytarabine for 24 hours, as detected by Annexin V staining (C) or by DNA content stained with DAPI (D) by flow cytometry. In cell lines with mutant DNMT3A more cells were in sub- $\mathrm{G}_{1}$ compared to cells with wild-type DNMT3A indicative of apoptosis $(* p<0.05, * * p<0.01, * * * p<0.001, * * * * p<0.0001$, two-way ANOVA with Tukey's post-hoc test from three replicates).

(E) Ex vivo cytarabine sensitivity in $5 D N M T 3 A$ wild-type and $4 D N M T 3 A$ R882 primary AML patient samples. All AMLs were $F L T 3^{I T D}: N P M 1^{c}(p=0.0502$, two-tailed Student's $t$-test).

(F, G) Apoptosis in U2OS cells lentivirally expressing wild-type (blue) or mutant (red) DNMT3A or empty vector control (black) and treated with cytarabine for 48 hours, as detected by Annexin V staining (F) or by DNA content stained with DAPI (fraction of cells in sub-G $\left.\mathrm{G}_{1}, \mathrm{G}\right)$ by flow cytometry $(* * * p<0.001$, **** $p<0.0001$, two-way ANOVA with Tukey's post-hoc test from three replicates).

(H) Similar steady-state growth rates of U2OS cells ectopically expressing wildtype (blue) or mutant (red) DNMT3A or empty vector control, as determined by methylene blue staining over time, relative to day 1 . Relative amount of retained methylene blue was assessed by absorbance $\mathrm{OD}_{570}$. 
A

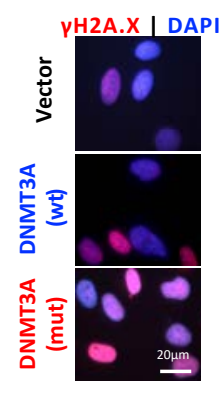

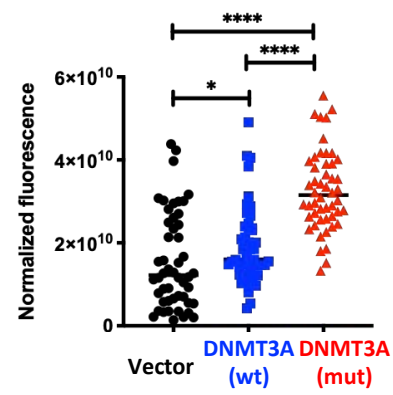

B

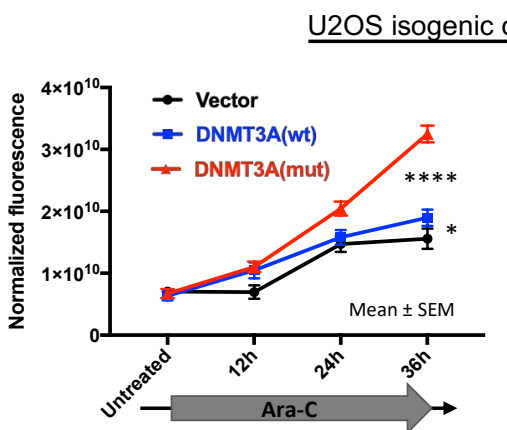

C

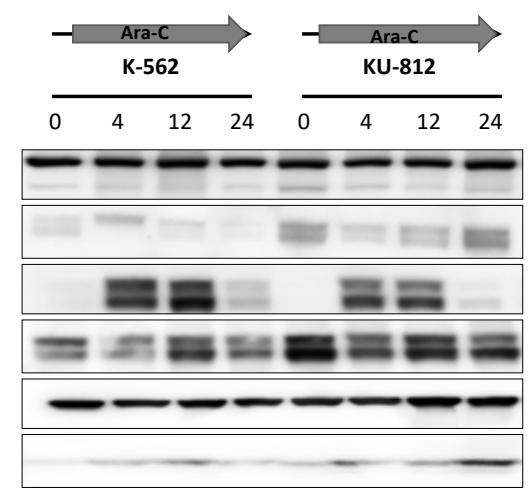

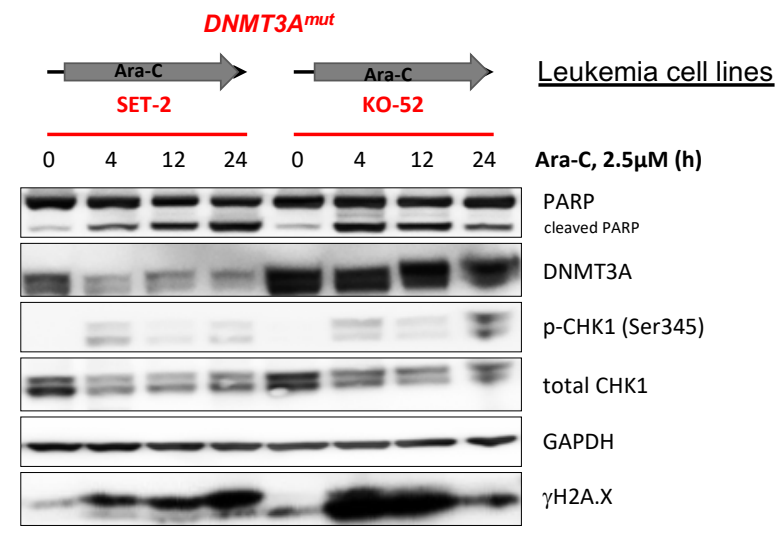

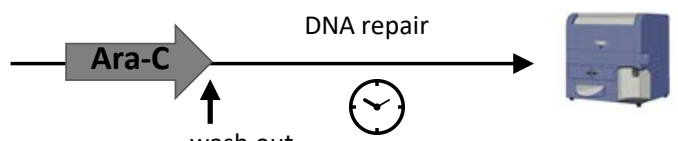

D
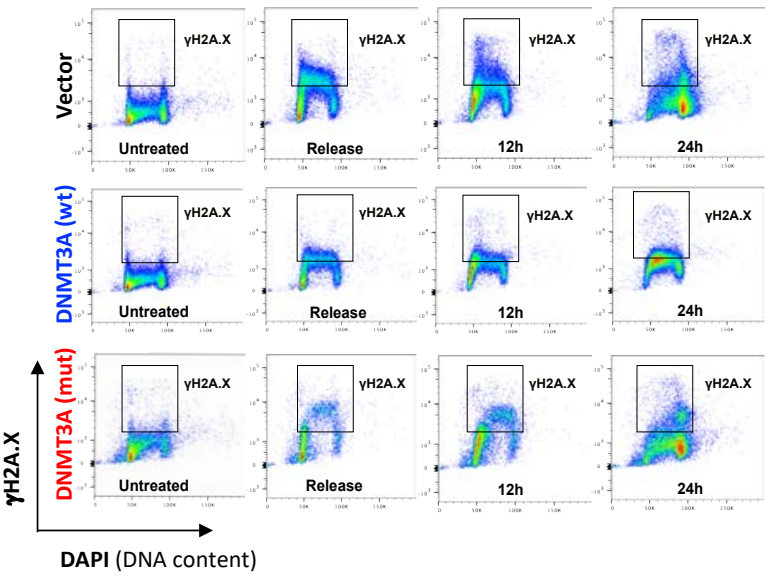

$\underline{\text { U2OS isogenic cells }}$

\section{E}

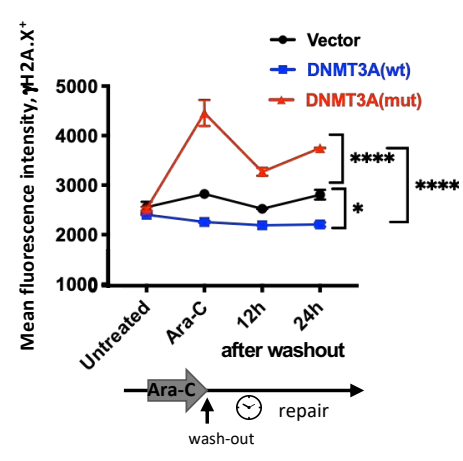


Supplementary Figure S2. Cells expressing mutant DNMT3A accumulate DNA damage due to incomplete DNA repair after cytarabine.

(A, B) Immunofluorescence staining of DNA damage marker $\gamma \mathrm{H} 2 \mathrm{~A} . \mathrm{X} 36$ hours (A) or over time at indicated timepoints (B) of treatment with $10 \mu \mathrm{M}$ cytarabine, in U2OS cells lentivirally expressing wild-type (blue) and mutant (red) DNMT3A or empty vector control (black). For quantitative analysis, at least 50 nuclei per condition were selected at random and background-corrected fluorescence intensity was measured using ImageJ software $(* p<0.05, * * * p<0.001, * * * * p<0.0001$, Mann-Whitney rank-sum test).

(C) Immunobloting analysis of DNA damage signaling and apoptosis at indicated timepoint after cytarabine exposure $(2.5 \mu \mathrm{M})$ in cell lines with wild-type (K-562, KU-812) and R882-mutant (SET-2, KO-52) DNMT3A.

(D, E) Persisting high levels of DNA damage in cells expressing mutant DNMT3A 12 and 24 hours after cytarabine wash-out. Flow cytometry scatter plots visualizing DNA damage marker $\gamma \mathrm{H} 2 \mathrm{~A} . \mathrm{X}$ across the cell cycle (DNA content stained by DAPI, D) and $\gamma$ H2A.X mean fluorescence intensity in cells surpassing DDNA damage threshold (E) in U2OS cells lentivirally overexpressing wild-type (blue) and mutant (red) DNMT3A or empty vector control (black), from two independent experiments performed in duplicate $(* p<0.05, * * * * p<0.0001$, Mann-Whitney rank-sum test). 
DNA damage $(\gamma \mathrm{H} 2 \mathrm{~A} . \mathrm{X})$

A

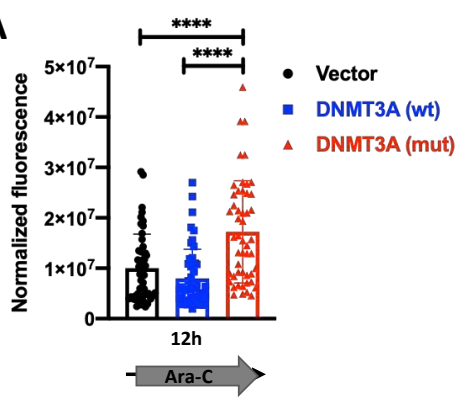

B

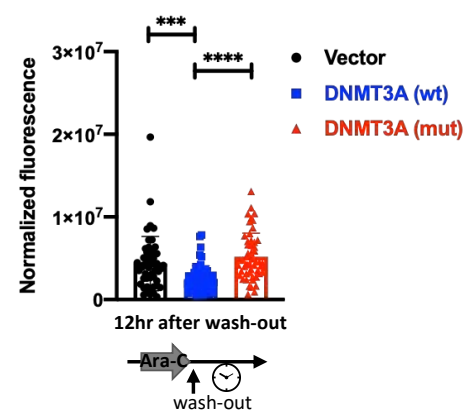

C
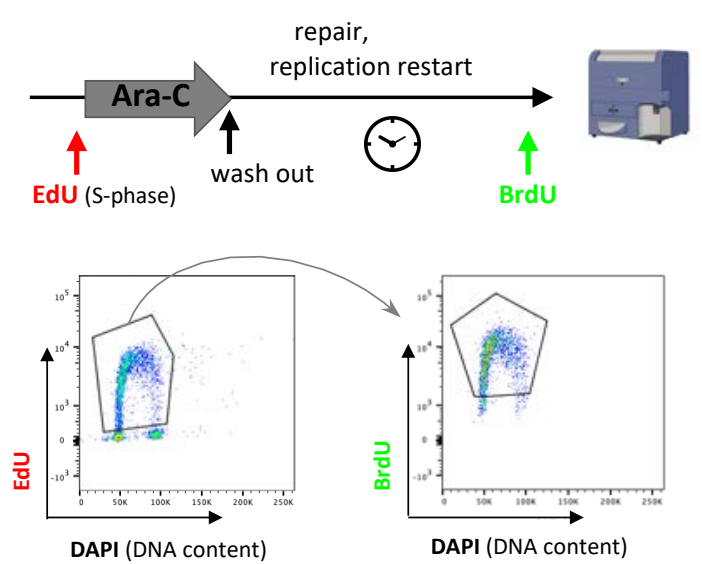

D

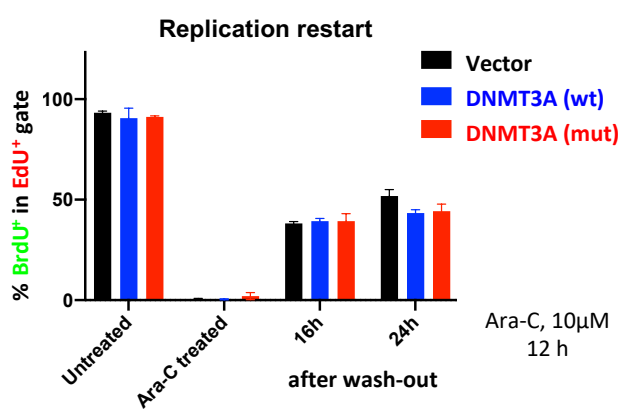




\section{Supplementary Figure S3. Cells expressing mutant DNMT3A accumulate DNA damage after cytarabine treatment yet are able to restart replication.}

(A, B) DNA damage measured by $\gamma \mathrm{H} 2 \mathrm{~A} . \mathrm{X}$ background-corrected fluorescence intensity after 12 hours of continuous cytarabine exposure (A) 12 hours following drug wash-out. At least 50 nuclei were quantified for each condition (*** $p<0.001, * * * * p<0.0001$, Mann-Whitney rank-sum test).

(C) Experimental workflow of the replication restart analysis by dual labeling with EdU (replicating cells pretreatment) and BrdU (at indicated time points after treatment or drug wash-out) and example flow cytometry scatter plots after exclusion of doublets and debris. Cell cycle was monitored by DNA content (DAPI). (D) Replication restart (fraction of $\mathrm{BrdU}^{+}$within $\mathrm{EdU}^{+}$population) in $\mathrm{U} 2 \mathrm{OS}$ cells expressing wild-type (blue) or mutant (red) $D N M T 3 A$ or empty vector control (black). No significant differences were detected between groups in three independent experiments each done in triplicate. 
bioRxiv preprint doi: https://doi.org/10.1101/2021.05.28.445639; this version posted May 28, 2021. The copyright holder for this preprint (which was not certified by peer review) is the author/funder. All rights reserved. No reuse allowed without permission.

A

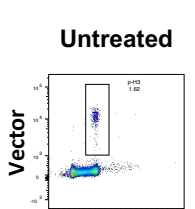

Ara-C, $10 \mu \mathrm{M}, 12 \mathrm{~h}$

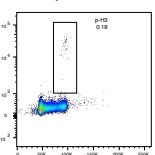

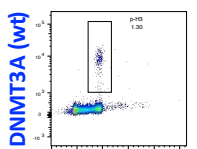

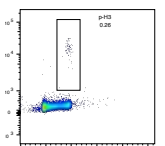

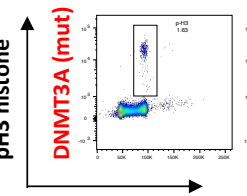

B

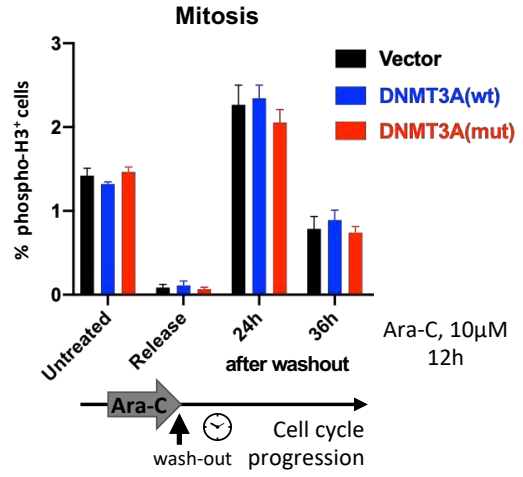

DAPI (DNA content)

C

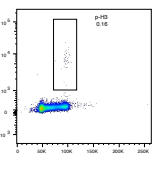

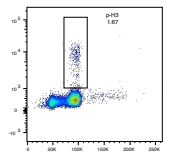
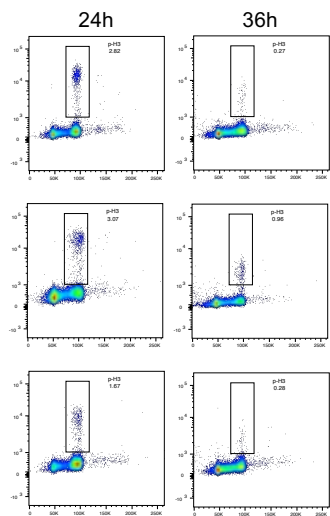

20

.

Cell cycle

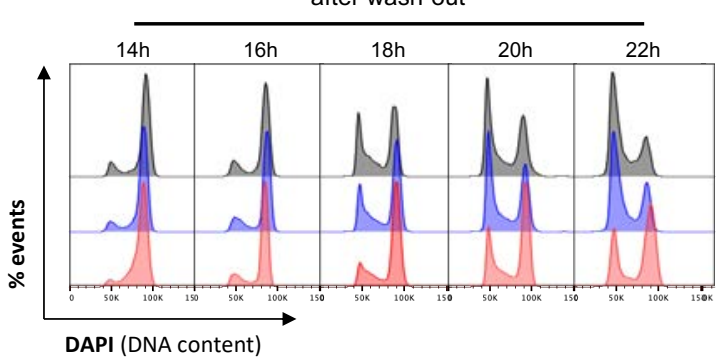

Ara-C, $10 \mu \mathrm{M}$

$12 \mathrm{~h}$

Vector

DNMT3A (wt)

DNMT3A (mut)

D

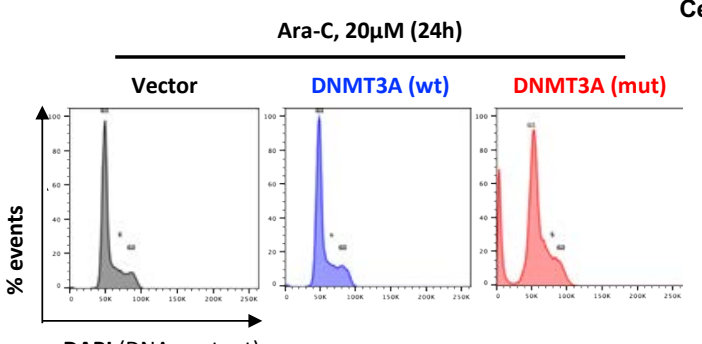

Cell cycle

DAPI (DNA content) 
Supplementary Figure S4. Delayed cell cycle progression in the cells expressing mutant DNMT3A after cytarabine treatment.

(A, B) Progression through mitosis after cytarabine treatment and removal was determined by mitotic marker phospho-Histone H3; DAPI was used to stain for DNA content (cell cycle). Representative flow cytometry scatter plots after gating out cell aggregates and debris (A) and fraction of cells undergoing mitosis (B) after cytarabine treatment and at indicated time points after drug wash-out, in U2OS cells with wild-type (blue) and mutant (red) $D N M T 3 A$ or empty vector control (black).

(C) Cell cycle profiles (by DNA content, stained by DAPI) in U2OS cells with wild-type (blue) and mutant (red) $D N M T 3 A$ or empty vector control (black) treated with cytarabine at indicated timepoints after wash-out.

(D) Flow cytometry-based cell cycle analysis in U2OS isogenic cells after cytarabine dose intensification $(20 \mu \mathrm{M}$ for 24 hours). Abnormal cell cycle profile (suppression of the $\mathrm{G}_{2}$ peak) in cells expressing mutant DNMT3A was observed. 
bioRxiv preprint doi: https://doi.org/10.1101/2021.05.28.445639; this version posted May 28, 2021. The copyright holder for this preprint (which was not certified by peer review) is the author/funder. All rights reserved. No reuse allowed without permission.

A

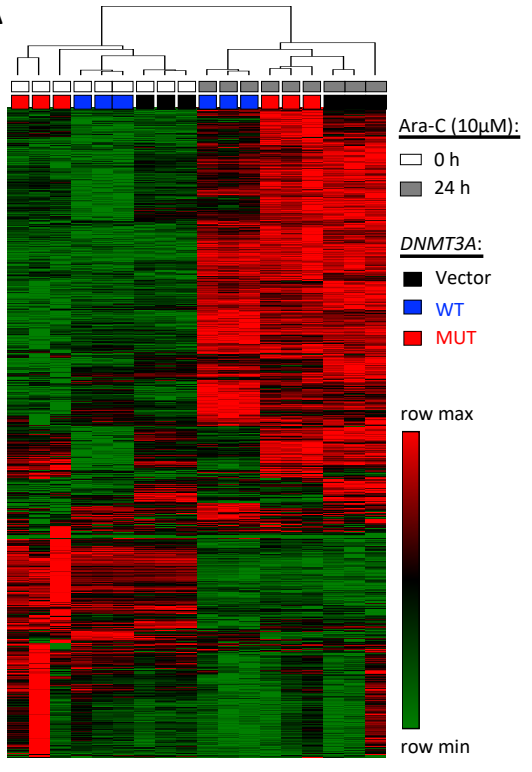

B

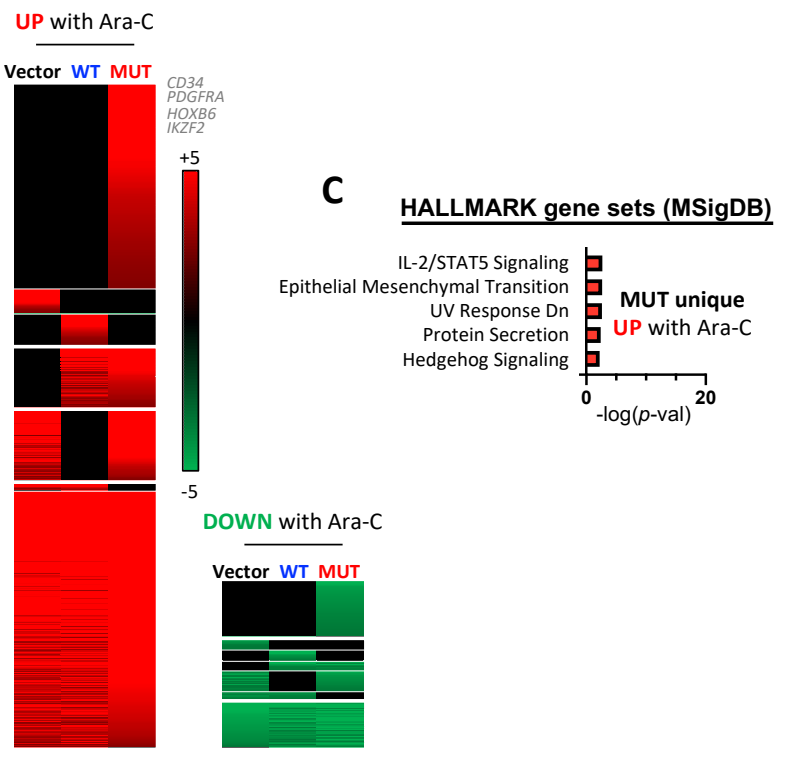

D

DNMT3A (mut) Ara-C vs DNMT3A (wt) Ara-C

GO: DNA Packaging Complex

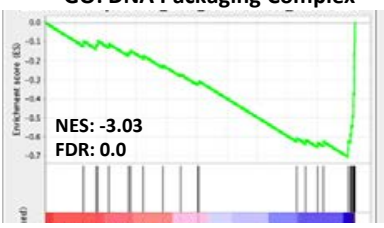

GO: Chromatin Assembly or

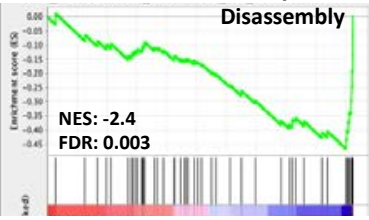

GO: Negative Regulation of Myeloid

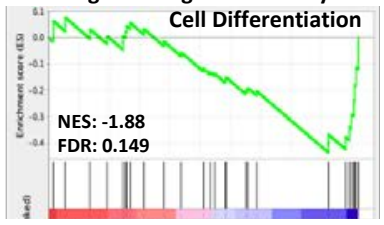




\section{Supplementary Figure S5. Gene expression profiling identifies pathways deregulated in cells expressing mutant DNMT3A after cytarabine treatment.}

(A) Unsupervised clustering on top 2000 most variable genes (by RNA-seq) demonstrates reproducibility between replicates and robust separation by treatment. U2OS cells lentivirally expressing wild-type (blue) or mutant (red) forms of DNMT3A or empty vector control (black) were treated with vehicle (PBS, white) or cytarabine (gray) for 24 hours, as biological triplicates.

(B) Heatmap of differentially expressed genes between Ara-C-treated and untreated cells, with genes commonly or uniquely regulated in each genotype grouped together. (C) Pathways of the HALLMARK collection (MSigDB) enriched among genes uniquely upregulated in $D N M T 3 A$ (mut) cells after cytarabine treatment (FDR $<0.1)$.

(D) Gene set enrichment analysis (GSEA) reveals negative enrichment of signatures related to chromatin organization and inhibition of myeloid differentiation in cells with $D N M T 3 A($ mut) compared to DNMT3A(wt), with cytarabine exposure. 


\section{Supplementary references}

1. Guryanova OA, Shank K, Spitzer B, et al. DNMT3A mutations promote anthracycline resistance in acute myeloid leukemia via impaired nucleosome remodeling. Nat Med. 2016;22(12):1488-1495.

2. de Hoon MJ, Imoto S, Nolan J, Miyano S. Open source clustering software. Bioinformatics. 2004;20(9):14531454.

3. Chen Y, Lun AT, Smyth GK. From reads to genes to pathways: differential expression analysis of RNA-Seq experiments using Rsubread and the edgeR quasi-likelihood pipeline. F1000Res. 2016;5:1438.

4. Chen EY, Tan CM, Kou Y, et al. Enrichr: interactive and collaborative HTML5 gene list enrichment analysis tool. BMC Bioinformatics. 2013;14:128.

5. Kuleshov MV, Jones MR, Rouillard AD, et al. Enrichr: a comprehensive gene set enrichment analysis web server 2016 update. Nucleic Acids Res. 2016;44(W1):W90-97.

6. Subramanian A, Tamayo P, Mootha VK, et al. Gene set enrichment analysis: a knowledge-based approach for interpreting genome-wide expression profiles. Proc Natl Acad Sci U S A. 2005;102(43):15545-15550.

7. Mootha VK, Lindgren CM, Eriksson KF, et al. PGC-1alpha-responsive genes involved in oxidative phosphorylation are coordinately downregulated in human diabetes. Nat Genet. 2003;34(3):267-273.

8. Liberzon A, Birger C, Thorvaldsdottir H, Ghandi M, Mesirov JP, Tamayo P. The Molecular Signatures Database (MSigDB) hallmark gene set collection. Cell Syst. 2015;1(6):417-425.

9. Kanehisa M, Goto S. KEGG: kyoto encyclopedia of genes and genomes. Nucleic Acids Res. 2000;28(1):27-30.

10. Snel B, Lehmann G, Bork P, Huynen MA. STRING: a web-server to retrieve and display the repeatedly occurring neighbourhood of a gene. Nucleic Acids Res. 2000;28(18):3442-3444.

11. von Mering C, Huynen M, Jaeggi D, Schmidt S, Bork P, Snel B. STRING: a database of predicted functional associations between proteins. Nucleic Acids Res. 2003;31(1):258-261.

12. Szklarczyk D, Gable AL, Lyon D, et al. STRING v11: protein-protein association networks with increased coverage, supporting functional discovery in genome-wide experimental datasets. Nucleic Acids Res. 2019;47(D1):D607-D613. 


\section{Supplementary tables}

Supplementary Table S1. Primary antibodies used for immunoblotting analyses.

\begin{tabular}{|c|c|c|c|}
\hline Antigen & Clone & Catalog Number & Manufacturer \\
\hline$\gamma$ H2A.X (S139) & $20 \mathrm{E} 3$ & $\# 9718$ & $\begin{array}{c}\text { Cell Signaling } \\
\text { Technology }\end{array}$ \\
\hline DNMT3A & D23G1 & $\# 3598$ & $\begin{array}{c}\text { Cell Signaling } \\
\text { Technology }\end{array}$ \\
\hline DNMT3A & C12 & sc-365769 & Santa Cruz Biotechnology \\
\hline p-Chk1 (S345) & $133 \mathrm{D} 3$ & $\# 2348$ & $\begin{array}{c}\text { Cell Signaling } \\
\text { Technology }\end{array}$ \\
\hline Total Chk1 & 2G1D5 & $\# 2360$ & $\begin{array}{c}\text { Cell Signaling } \\
\text { Technology }\end{array}$ \\
\hline PARP & Rabbit polyclonal & $\# 9542$ & $\begin{array}{c}\text { Cell Signaling } \\
\text { Technology }\end{array}$ \\
\hline GAPDH & 14 C10 & $\# 2118$ & $\begin{array}{c}\text { Cell Signaling } \\
\text { Technology }\end{array}$ \\
\hline p-p53 (S15) & Rabbit polyclonal & $\# 9284$ & $\begin{array}{c}\text { Cell Signaling } \\
\text { Technology }\end{array}$ \\
\hline Total p53 & DO-1 & sc-126 & Santa Cruz Biotechnology \\
\hline PARP1 & F-2 & Sc-8007 & Santa Cruz Biotechnology \\
\hline
\end{tabular}

Supplementary Table S2. Primary antibodies used for intracellular flow cytometry.

\begin{tabular}{|c|c|c|c|}
\hline Antigen (fluorophore) & Clone & Catalog Number & Manufacturer \\
\hline BrdU (FITC) & BU20A & $11-5071-42$ & Thermo Fischer Scientific \\
\hline $\begin{array}{c}\gamma \text { H2A.X } \\
\text { (AlexaFluor 647) }\end{array}$ & $2 \mathrm{~F} 3$ & 613408 & Biolegend \\
\hline $\begin{array}{c}\text { p-Histone H3 (S28) } \\
\text { (AlexaFluor 647) }\end{array}$ & HTA28 (RUO) & 558217 & BD Biosciences \\
\hline BrdU (AlexaFluor 488) & MoBU-1 & B35130 & Invitrogen \\
\hline
\end{tabular}

Supplementary Table S3. Primary antibodies used for immunofluorescence.

\begin{tabular}{|c|c|c|c|}
\hline Antigen & Clone & Catalog Number & Manufacturer \\
\hline$\gamma$ H2A.X & JBW301 & $05-636$ & Millipore \\
\hline PCNA & PC10 & sc-56 & Santa Cruz Biotechnology \\
\hline p-RPA2 (S33) & Rabbit polyclonal & NB100-544 & Novus Biologicals \\
\hline BrdU & BU-1 & MA3-071 & Invitrogen \\
\hline BrdU & MoBU-1 & B35128 & Invitrogen \\
\hline Lamin B1 & Rabbit polyclonal & $\mathrm{ab} 16048$ & Abcam \\
\hline
\end{tabular}

